\title{
THE HEATING OF DUST IN STARBURST GALAXIES: THE CONTRIBUTION OF THE NONIONIZING RADIATION
}

\author{
Daniela Calzetti, ${ }^{1}$ Ralph C. Bohlin, and AnNe L. Kinney ${ }^{1,2}$ \\ Space Telescope Science Institute, 3700 San Martin Drive, Baltimore, MD 21218 \\ Thaisa STORCHI-BERgManN ${ }^{2}$ \\ Instituto di Fisica, Universidade Federal Rio Grande do Sul, Brazil \\ AND \\ Timothy M. HeCKMAN \\ Department of Physics and Astronomy, The Johns Hopkins University \\ Received 1994 June 3; accepted 1994 September 30
}

\begin{abstract}
The IUE UV and optical spectra and the far-infrared (FIR) IRAS flux densities of a sample of starburst and blue compact galaxies are used to investigate the relationship between dust obscuration and dust emission. The amount of dust obscuration at UV wavelengths correlates with the FIR-to-blue ratio; and an analysis of the correlation indicates that not only the ionizing but also the nonionizing radiation contribute to the FIR emission. The amount of UV and optical energy lost to dust obscuration accounts for most of the cool dust FIR emission and for about $70 \%$ of the warm dust FIR emission The remaining $30 \%$ of the warm dust FIR flux is probably due to dust emission from regions of star formation which are embedded in opaque giant molecular clouds and do not contribute to the integrated UV and optical spectrum.

The use of the FIR emission as an indicator of high-mass star formation rate in star-forming galaxies can be problematic, since the contribution to the FIR flux from cool dust emission heated by relatively old stars is nonnegligible $(\sim 30 \%)$.
\end{abstract}

Subject headings: dust: extinction — galaxies: ISM — galaxies: starburst - infrared: galaxies ultraviolet: galaxies

\section{INTRODUCTION}

The 10-120 $\mu \mathrm{m}$ IRAS emission from galaxies without nuclear nonthermal activity is due to dust heated by the galaxy radiation field (e.g., Rieke et al. 1980; de Jong et al. 1984; see also Young et al. 1989). Both the young, massive stars and the old stars contributing to the general interstellar radiation field (GISRF) can heat the dust which emits in the IRAS bands (Helou 1986; Rowan-Robinson \& Crawford 1989). The dust heated by these two classes of stars produces far-infrared (FIR) emission of different strengths at different wavelengths.

Various models for the composition of the interstellar dust have been proposed to explain the UV and optical extinction and the infrared emission of dust (e.g., Puget \& Léger 1989; Désert, Boulanger, \& Puget 1990; see also Draine \& Anderson 1985 and, for a model for our Galaxy, Cox, Krügel, \& Mezger 1986). Without discussing the details of any specific model, we provide some general outlines which will be useful in this work. Two components, large molecules (sizes $\sim 10 \AA$ ) and small grains (sizes $\sim 50-100 \AA$ ), can be introduced to explain the IRAS emission at 12 and $25 \mu \mathrm{m}$ and part of the $60 \mu \mathrm{m}$ emission. The two components are not in thermal equilibrium with the surrounding environment but are heated by single-photon absorption processes to temperatures ranging from hundreds to about 1000 K (Sellgren 1984; Draine \& Anderson 1985; see

\footnotetext{
${ }^{1}$ Visiting Astronomers, Kitt Peak National Observatory, National Optical Astronomy Observatories, which is operated by AURA, Inc., under a cooperative agreement with the National Science Foundation.

2 Visiting Astronomers Cerro Tololo Inter-American Observatory, National Optical Astronomy Observatories, which is operated by AURA, Inc., under a cooperative agreement with the National Science Foundation.
}

also Andriesse 1978). Désert et al. (1990) propose that small grains, of carbonaceous nature, and large molecules, possibly polycyclic aromatic carbons (PAHs) (Léger \& Puget 1984), are responsible for the $2200 \AA$ bump and the far-UV nonlinear rise of the extinction curve, respectively. A third dust component of large silicate grains (sizes $>100 \AA$ ), coated or uncoated (e.g., Mathis \& Whiffen 1989; Duley, Jones, \& Williams 1989; Chlewicki \& Greenberg 1990), and in thermal equilibrium with the surrounding environment, is required to account for a large fraction of the $60 \mu \mathrm{m}$ and most of the $100 \mu \mathrm{m}$ IRAS emission (the FIR emission, as defined by Helou, Soifer, \& RowanRobinson 1985; see also Helou et al. 1988). In the presence of the GISRF, the large grains have typical temperatures $T \sim 15-20 \mathrm{~K}$ and produce the so-called cool dust emission, which peaks at a wavelength $\lambda>100 \mu \mathrm{m}$. FIR emission from Galactic cirrus provides an example of cool dust emission (Low et al. 1984; Rowan-Robinson \& Crawford 1987; de Jong $\&$ Brink 1987). In the presence of massive stars, the large grains are heated to temperatures $T \sim 30-50 \mathrm{~K}$ and produce the socalled warm dust emission, which peaks around $60 \mu \mathrm{m}$. Ionizing photons from massive stars also destroy large molecules, decreasing the intensity of the $12 \mu \mathrm{m}$ emission (Boulanger et al. 1988).

Though this simplified three-component picture has been successful in explaining the distribution of nonactive galaxies along the IRAS color-color sequence (Pajot et al. 1986; Helou 1986; see Boulanger et al. 1988), doubts have been cast on the relative importance of the two types of heating sources in contributing to the emission detected in the IRAS bands: there is an ongoing debate on whether the young massive stellar component or the relatively old stellar population is the main 
source of dust heating in quiescent spirals (see, e.g., Devereux \& Young 1991, 1992, 1993; Helou 1986; Lonsdale-Persson \& Helou 1987, hereafter LH; Sauvage \& Thuan 1992). If the young massive stars are responsible for the heating of the dust emitting in the IRAS bands at 60 and $100 \mu \mathrm{m}$, then the use of the FIR emission as an indicator of the high-mass star formation rate (SFR) in the galaxy may be possible (Young et al. 1989; Devereux \& Young 1991).

The IRAS FIR dust emission is probably a good indicator of high-mass SFR in galaxies with ongoing large star formation activity, since the young $\mathrm{OB}$ associations $\left(M \gtrsim 5 M_{\odot}\right)$ may contribute to a large fraction of the radiation field (see the discussion in Hunter et al. 1989a for a sample of irregular galaxies). However, testing whether the FIR emission is a measure of the SFR in these galaxies means understanding the relative contribution of the massive stars and the GISRF to the dust heating which produces the FIR emission. The problem is additionally complicated; in fact, while the ionizing radiation is mostly absorbed by the dust and the gas, and little emerges from the galaxy as emission lines, the absorption of the nonionizing radiation depends on the amount of dust in front of the stars, and the net contribution to the FIR emission increases with the quantity of dust in front of the stars (e.g., Belfort, Mochkovitch \& Dennefeld 1987). Understanding the role of the nonionizing radiation in the dust heating process is a key to understanding the nature of dust emission in starforming galaxies.

In normal (nonstarbursting) spiral galaxies, different approaches have been taken to estimate the fraction of cool dust emission in the IRAS bands. Chini et al. (1986), de Jong \& Brink (1987), and LH have introduced a two-component model with single-valued temperature to describe the FIR emission. As remarked by the authors who introduced it, the model is an oversimplification of the reality, and the two temperatures should not be taken literally. Quoting LH, "There is not likely to be a sharp boundary in ... temperature." However, the model has been shown to give a qualitatively interesting description of the FIR emission from galaxies. The two components, cool and warm dust emission, are present in different proportions in different Hubble types, with the cool component ranging from $\sim 86 \%$ in Sa galaxies to $\sim 70 \%$ in $\mathrm{Sc}$ galaxies (Sauvage \& Thaun 1992; see, however, Devereux \& Young 1991 for a different point of view). Buat \& Deharveng (1988), Boulanger \& Pérault (1988), and Hunter et al. (1989a) have pointed out the importance of the nonionizing UV radiation as a contributor to the GISRF producing the cool dust emission. In particular, Buat \& Deharveng (1988) find that the UV emission at $\lambda \sim 2000 \AA$ has a tighter correlation with the FIR cool dust emission than with the FIR warm dust emission. $\mathrm{Xu}$ (1990) shows that in normal galaxies the nonionizing UV radiation can explain up to $76 \%$ of the cool dust emission.

A detailed estimation of the contribution of the nonionizing radiation to the FIR emission is hampered by two factors: (1) the lack of suitable UV spectral information and (2) the lack of an appropriate obscuration curve to be applied to the UV and optical stellar continua.

The lack of suitable UV data is linked primarily to the fact that the UV and optical spectral information is usually obtained in relatively small apertures, while infrared data are obtained with large apertures, making the comparison between the two sets of measures difficult. The $10^{\prime \prime} \times 20^{\prime \prime} I U E$ UV aperture is one of the largest available. The $I R A S$ infrared data are obtained in apertures which range from $0.75 \times 4^{\prime} .5$ for the 12 $\mu \mathrm{m}$ beam to $3^{\prime} \times 5^{\prime}$ for the $100 \mu \mathrm{m}$ beam. A way out for this problem is working with galaxies which have most of their light concentrated toward the central regions, e.g., nuclear starforming galaxies. Our sample fulfills this requirement, and the galaxies for which we have evaluated the energetic balance between dust absorption and emission have $20 \%$ or more of their blue light within the UV-optical aperture.

Though the problem of applying standard interstellar extinction curves to the integrated stellar continua of galaxies has been already recognized (e.g., Keel 1993; Fanelli, O'Connell, \& Thuan 1988), quantifying the problem has been difficult. Only recently an "extragalactic obscuration curve" has been derived (see Fig. 1; Calzetti et al. 1994, hereafter CKS; Kinney et al. 1994). This curve is used here to estimate the amount of UV and optical energy lost to dust obscuration and converted into infrared emission.

The plan is to estimate the contribution of the massive stars and the GISRF to the infrared dust emission for a sample of starburst and blue compact galaxies. The purpose is (1) to understand which fraction of the cool dust emission can be accounted for when the UV and optical spectra are corrected for dust obscuration using the CKS curve and (2) to study whether the FIR emission is a viable indicator of high-mass SFR in star-forming galaxies.

The sample, the adopted UV and optical obscuration curve, and the criteria to decompose the $I R A S$ flux densities into the cool and warm dust emission are described in $\S 2$. In $\S 3$ the relation between the UV dust obscuration and the "infrared activity," defined as the FIR-to-blue luminosity ratio, is investigated. In $\S 4$ we give a detailed estimation of the fraction of cool and warm dust emission accounted for by our UV and optical spectra. The summary and the conclusions are given in $\S 5$.

Our investigation will concentrate on the infrared emission due to large grains in thermal equilibrium with the environment, therefore on the characteristics of the emission at 60 and $100 \mu \mathrm{m}$. The emission from nonequilibrium small grains/large molecules, mainly at 12 and $25 \mu \mathrm{m}$, will not be discussed here, except for a brief mention in $\S 3.2$.

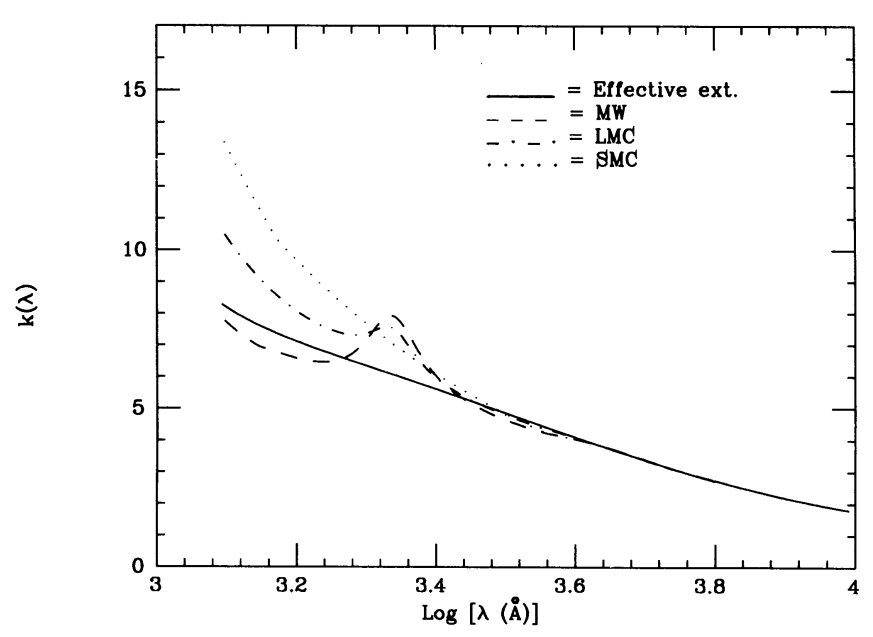

FIG. 1.-The obscuration curve for external galaxies, eq. (3), as a function of the wavelength (solid line). The curve is plotted with normalization $E(B-V)=1$ and $k(V)=A(V) / E(B-V)=3.1$. For comparison, the Milky Way, the Large Magellanic Cloud, and the Small Magellanic Cloud interstellar extinction curves are shown on the same scale. 


\section{THE SAMPLE}

Of the 143 galaxies contained in the Atlas of Ultraviolet Spectra of Star-Forming Galaxies (Kinney et al. 1993), 94 have ongoing starburst activity in their nucleus. Particular care has been taken in removing from the set the galaxies showing evidence for mixed activity (e.g., starburst + Seyfert or starburst + low-ionization nuclear emission-line region [LINER]), since the infrared emission may be contaminated by emission from the nuclear nonthermal source. Hot spot galaxies have been excluded as well. Thus, the sample is reduced to 80 nuclear starburst or blue compact galaxies.

Though galaxies with evidence for nonthermal activity in their nucleus have been removed from the sample, the possibility for a hidden active galactic nucleus (AGN) in some of the 80 galaxies cannot be excluded a priori. Being shrouded in dust, the AGN would contribute to the infrared emission but not to the UV and optical radiation. However, since the starburst activity appears to dominate the emission in all the 80 galaxies, as inferred from the emission line ratios, we will assume that an AGN, if present, has a very low luminosity in comparison to the burst of star formation.

\subsection{The Ultraviolet and Optical Data}

The 80 galaxies have UV IUE spectra which cover the wavelength range $1200-3200 \AA$ (or 1200-2000 $\AA$ ) and are taken in an aperture of $20^{\prime \prime} \times 10^{\prime \prime}$ (hereafter sample S1). Optical spectra are available for a subsample of 36 galaxies in $S 1$; this subsample will be indicated as S2. The optical spectra cover the wavelength range $3200-8000 \AA$ and were obtained in an IUEmatched aperture. The main characteristics of the UV and optical emission of the $\mathrm{S} 2$ galaxies can be found in CKS, where $\mathrm{S} 2$ is used to derive the dust obscuration properties in starburst galaxies.

From the original sample $\mathrm{S} 1$, the following objects are removed: NGC 2820A, UGC 6448, UGC $8315 \mathrm{~N}$, and Mrk 309 , because of the low signal-to-noise ratio of their IUE spectra; NGC 4853 and NGC 5102, because their optical spectrum is red and does not show any of the nebular emission lines typical of star-formation activity; and $1050+04$, because it is located in one of the areas of the sky not surveyed by IRAS. NGC 5457 (M101) is also removed, because it is an extended galaxy (angular diameter $\sim 30^{\prime}$ ) with many OB associations in its spiral arms, making any comparison between the nuclear UV emission and the more extended infrared emission meaningless.

The UV spectra of the remaining 72 galaxies (see the list in Table 1) have been fitted with a power law in the wavelength range $1250-2600 \AA$ (or $1250-2000 \AA$, when only the shortwavelength $I U E$ spectrum is available):

$$
F(\lambda)=F_{0} \lambda^{\beta}
$$

where $\beta$ is the UV spectral index. According to CKS, the spectral index $\beta$ is a good indicator of dust obscuration in these galaxies. A linear relation exists between $\beta$ and the color excess $E_{l}(B-V)$ obtained from the ratio of the Balmer emission lines $\mathrm{H} \alpha / \mathrm{H} \beta$ (see CKS for details):

$$
\beta=1.88 E_{l}(B-V)-1.71 \text {. }
$$

For $E_{l}(B-V)=0, \beta(0)=-1.71$, which is a little redder than the theoretical value $\beta(0) \sim-2.3$ obtained from models of unobscured spectra of massive star populations (Leitherer \& Heckman 1994). The discrepancy can be understood if there is contamination of some of the UV spectra from stellar populations older than a few times $10^{7}$ yr. Given the large $I U E$ aperture, such contamination can be expected but does not represent the source of the correlation in equation (2) (see CKS). In equation (2), increasing amounts of dust obscuration, i.e., increasing values of the color excess, correspond to increasing values of the spectral index $\beta$.

A detailed analysis will be performed on the galaxies for which both UV and optical spectra are available (sample S2; see the list in Table 3). For these galaxies, an obscuration curve is given (CKS):

$$
k(x)=-2.155+3.678 x-0.483 x^{2}+0.027 x^{3},
$$

where $x=1 / \lambda\left(\mu \mathrm{m}^{-1}\right)$. The curve is normalized to $E(B-V)=1$ and $k(V)=A(V) / E(B-V)=3.1$ (see Fig. 1). Equation (3) can be used as a standard extinction curve to deredden the emergent stellar continuum spectra:

$$
F^{i}(\lambda)=F^{\text {obs }}(\lambda) 10^{0.4 E_{c}(B-V) k(\lambda)},
$$

where $F^{i}(\lambda)$ is the intrinsic galaxy spectrum, $F^{\text {obs }}(\lambda)$ is the emergent spectrum, and $E_{c}(B-V)$ is the color excess of the stellar continuum. CKS, Kinney et al. (1994), and Stochi-Bergmann, Calzetti, \& Kinney (1994) verified, on the basis of observational data and using two different methods, that the dust obscuration of the stellar continuum for the galaxies in $\mathbf{S} 2$ is given by:

$$
E_{c}(B-V)=0.5 E_{l}(B-V),
$$

where $E_{l}(B-V)$ is the color excess derived from the Balmer emission lines. The discrepancy between $E_{l}(B-V)$ and $E_{c}(B-V)$ is attributed to the fact that high-mass ionizing stars are associated with dustier regions than nonionizing stars. Therefore, the nebular emission lines, due to ionization from massive stars, show a larger amount of dust obscuration than the UV and optical stellar continuum, which is due also to the general stellar population.

Equation (4) assumes that the dust responsible for the obscuration is located in a foreground screen. This approximation may seem inadequate for starburst nuclei, where hot stars can be heavily embedded in dust. However, from the analysis of the galaxies in S2, CKS found that the foreground screen model is compatible with the observational data, while other models, among them the homogeneous mixture of dust and stars, fail to reproduce the data.

Equations (3)-(5) are used to deredden the UV and optical spectra of the galaxies in sample $\mathrm{S} 2$ and to calculate the amount of nonionizing energy $\left[\delta F_{(912-8000)}=F^{i}-F^{\text {obs }}\right]$ which has been absorbed by dust and converted into infrared emission (see Fig. 2).

In order to minimize the effect of the aperture mismatch between IUE and IRAS, and to make the comparison between UV-optical and infrared data more consistent, S2 does not include the galaxies which have less than $20 \%$ of the blue luminosity within the $I U E$-optical aperture. This selection criterion excludes the three most extended galaxies in the sample, NGC 1313, NGC 5236, and NGC 7793. Among the remaining 32 galaxies, 18 have $60 \%$ or more of the total blue light within the IUE-optical aperture (second column of Table 3). The blue luminosity in the $I U E$ aperture has been evaluated by convolving the Johnson $B$ filter response curve with our optical spectra; the normalization to the total $B$ magnitude has been obtained by setting the ratio $L_{B(I U E)} / L_{B}=1$ for the four galaxies whose angular diameters are small enough to be com- 
TABLE 1

OBSERVED QUANTITIES FOR SAMPLE S1

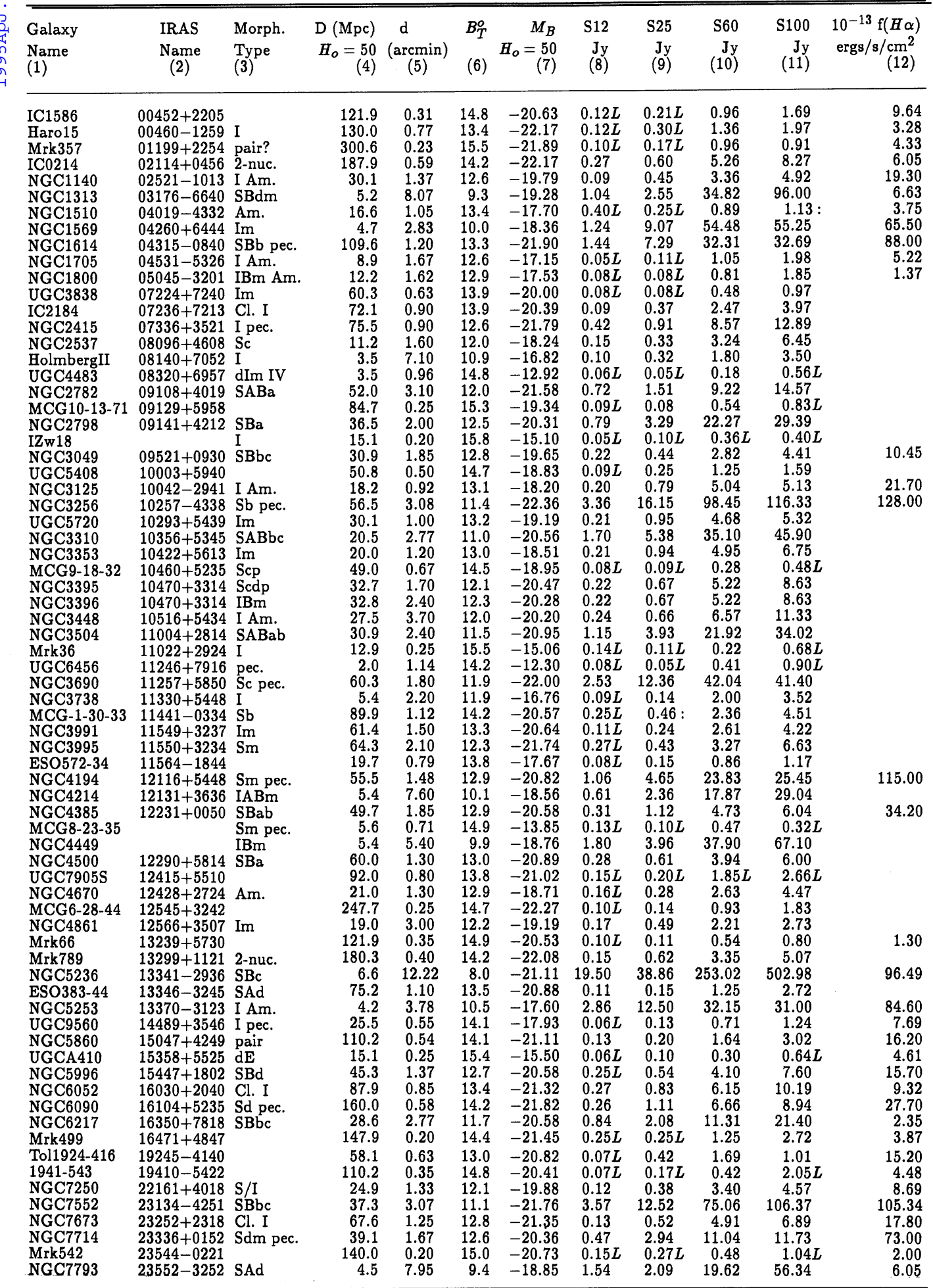

Notes - The IRAS names (col. [2]) are from the IRAS Point Source Catalog or from the IRAS Faint Source Catalog, NGC 4449 has no IRAS name because it was an Additional Observation (see Young et al. 1989); I Zw 18 and MCG 8-23-35 are not reported in the two catalogs, so they have no IRAS names. The morphological classification (col. [3]) is from the Nasa Extragalactic Database (NED). The distance $D$ (col. [4]) is from Kinney et al. 1993, where a Hubble constant $H_{0}=50 \mathrm{~km} \mathrm{~s}^{-1}$ $\mathrm{Mpc}^{-1}$ is assumed. The apparent diameter $d$, in arcminutes, (col. [5]) is the rms of the maximum and minimum diameters of the galaxy. The value of $B_{T}^{0}$ (col. [6]), or the value of the blue magnitude when $B_{T}^{0}$ is not available, is from RC3. $M_{B}$ (col. [7]) is the absolute blue magnitude derived from the total blue magnitude of the galaxy. S12, S25, S60, S100 (cols. [8], [9], [10], [11]) are the $I R A S$ flux densities (see $\S 2.2$ ); an L following the IRAS flux denotes an upper limit; a colon denotes a moderate quality flux density (see Fullmer \& Lonsdale 1989). The $\mathbf{H} \alpha$ emission line flux $f(\mathbf{H} \alpha)$, (col. [12]) is derived from our optical spectra, obtained from the central 200 square arcsec of the galaxy. The $\mathbf{H} \alpha$ flux has been extinction corrected using a Seaton curve (1979) and the color excess derived in Calzetti, Kinney, \& Storchi-Bergmann 1994. 


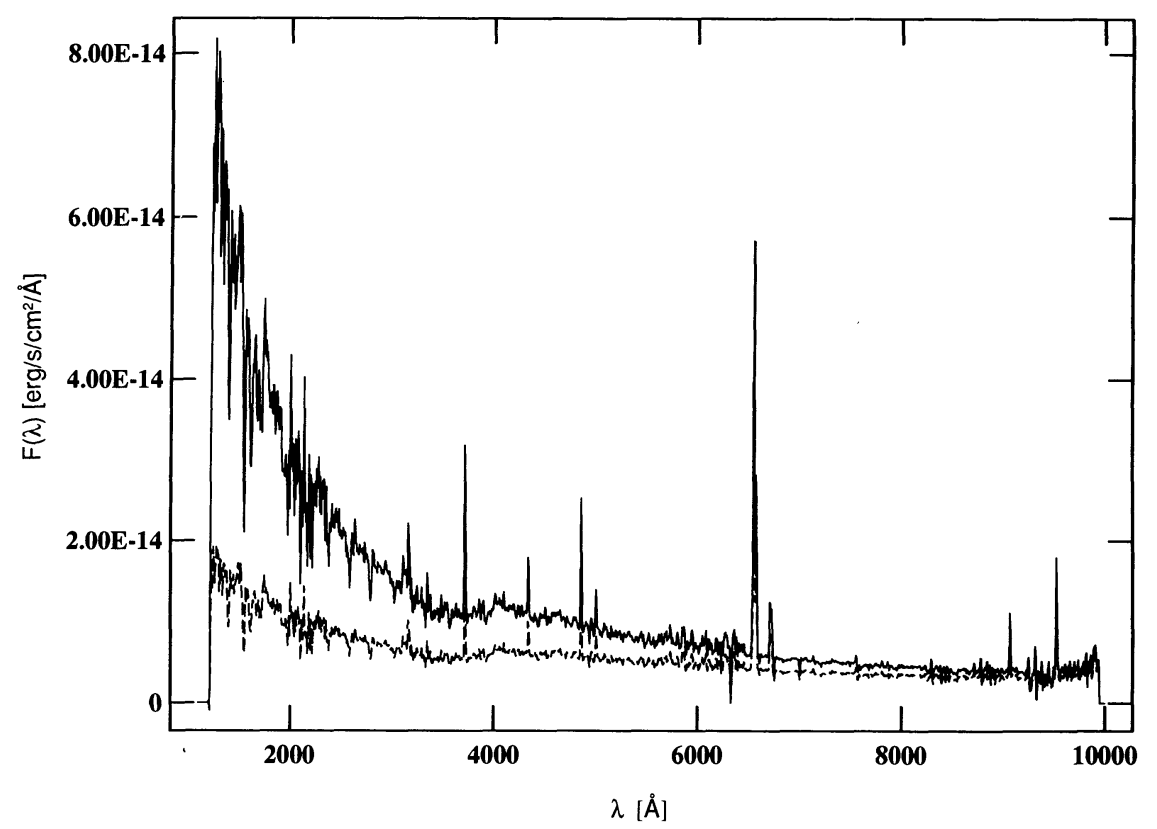

Fig. 2.-The observed and obscuration-corrected spectra of one of the sample's galaxies, NGC 438j, in the wavelength range 1200-10,000 $\AA$. The observed spectrum has been corrected for obscuration using eq. (3) and the color excess $E_{c}(B-V)$ reported in Table 3. The area between the two curves is the amount of nonionizing energy lost to dust obscuration in the region $1200-10,000 \AA$. The reddened and obscuration-corrected spectra is the wavelength region $912-1200 \AA$ (not shown here) are reconstructed by extrapolation (see $\S 4)$.

pletely included within the IUE aperture, namely, Mrk 357, Mrk 499, UGCA 410, and Mrk 542 (see fifth column of Table 1).

\subsection{The Far-Infrared Data}

The infrared fluxes at $12,25,60$, and $100 \mu \mathrm{m}$ for the galaxies in S1 were collected from three sources: the Catalogued Galaxies and Quasars Observed in the IRAS Survey (Fullmer \& Lonsdale 1989), the IRAS Faint Source Catalog, and, for the galaxies with optical sizes larger than $8^{\prime}$, the Catalog of $I R A S$ Observations of Large Optical Galaxies (Rice et al. 1988; see also Rice 1993). Total fluxes for galaxies flagged as extended in the Fullmer \& Lonsdale catalog were obtained by running ADDSCAN/SCANPI at IPAC (see the description in Young et al. 1989). ADDSCAN/SCANPI was also used to set upper limits on IRAS fluxes for two undetected galaxies (I Zw 18 and MCG 8-23-25) and to check for extended emission galaxies with optical sizes larger than 1.5 (the size of the $60 \mu \mathrm{m}$ beam). As a consistency check, the results of our analysis were compared with published data on galaxies with extended emission (Young et al. 1989; Hunter et al. 1989a; Soifer et al. 1989).

Our analysis concentrates on the FIR thermal emission from large dust grains, which dominates the 60 and $100 \mu \mathrm{m} \mathrm{IRAS}$ bands. Then, a galaxy is considered "detected" when both the $60 \mu \mathrm{m}$ and $100 \mu \mathrm{m}$ IRAS bands have measurements. Of the 72 galaxies in S1, 61 are detected, nine have upper limits in the 100 $\mu \mathrm{m}$ band, and two have upper limits in both the 60 and $100 \mu \mathrm{m}$ bands.

The 72 galaxies are listed in Table 1, together with their IRAS names, the morphological type, the distance $D$, the apparent diameter $d$, the $B_{T}^{0}$ magnitude (when available) or other integrated blue magnitudes from the RC3, the absolute blue magnitude $\left(H_{0}=50 \mathrm{~km} \mathrm{~s}^{-1} \mathrm{Mpc}^{-1}\right.$ is assumed), the infrared fluxes at $12,25,60$, and $100 \mu \mathrm{m}$, and the dereddened $\mathrm{H} \alpha$ flux, when the optical spectra are available. Like all the quantities derived from the UV or the optical spectra, the $\mathrm{H} \alpha$ flux refers to observations taken in a 200 square arcsec aperture, while the infrared data cover the entire galaxy. We will discuss the problem of the apperture mismatch later.

In Table 1, there are four galaxy pairs unresolved by IRAS: NGC 3395/NGC 3396, NGC 3690/IC 694, NGC 3994/NGC 3995, UGC 7905S/UGC 7905N. The infrared fluxes of the components for the first pair have been separated by assuming proportionality between the FIR emission and the $\mathrm{H} \alpha$ emission and by using the total $\mathrm{H} \alpha$ luminosities of Kennicutt et al. (1987). The second pair has been resolved using the analysis by Joy et al. (1989). The infrared components of the third pair have been separated according to Surace et al. (1993). For the fourth pair, the IRAS fluxes are assumed as upper limits on the FIR fluxes of UGC 7905S.

Although NGC 1313 is classified as an H II galaxy (see references in Kinney et al. 1993), there are a few peculiarities which suggest that the galaxy is a more complex object: (1) its flux density profiles at 12,25 , and $60 \mu \mathrm{m}$ show two peaks of emission (Rice et al. 1988), unlike other extended starburst and hot spot galaxies in Rice et al.'s atlas; (2) the highly absorbed nuclear X-ray spectrum is compatible with a low-luminosity Seyfert nucleus (Fabbiano \& Trinchieri 1987). Indeed, NGC 1313 shows a behavior which is slightly different from the other galaxies of the sample (see below). This characteristic will be taken into account in the following analysis.

In the present discussion, the FIR flux is defined as (Helou et al. 1988)

$$
\text { FIR }=1.26 \times 10^{-11}\left(2.58 S_{60}+S_{100}\right),
$$

and the FIR luminosity as

$$
L_{\mathrm{IR}}=4 \pi D^{2} \mathrm{FIR}
$$

where FIR is expressed in ergs $\mathrm{cm}^{-2} \mathrm{~s}^{-1}, D$ is the distance of the galaxy, and $S_{60}$ and $S_{100}$, in jansky, are the observed IR $A S$ fluxes at 60 and $100 \mu \mathrm{m}$. FIR and $L_{\mathrm{IR}}$ define the infrared flux and luminosity in the range $40-120 \mu \mathrm{m}$ (Helou et al. 1988) and 
represent a fraction of the total infrared emission $F(1-1000 \mu \mathrm{m})$ from a galaxy.

Following previous works (e.g., Chini et al. 1986; de Jong \& Brink 1987; LH), the FIR fluxes can be decomposed into the two contributions from the cool and warm dust emission, FIR $=$ FIR $^{c}+$ FIR $^{w}$. The two-component model is introduced here to provide a handy term of comparison for the amount of energy from the massive stars and the GISRF absorbed by dust and reemitted in the FIR. In $\S 3$ and in the first part of $\S 4$ we implicitly assume that the ionizing stellar radiation is associated with the massive stars and the warm dust emission, and the nonionizing stellar radiation is entirely associated with the GISRF and the cool dust emission. The contribution to the warm dust emission from the nonionizing radiation emitted by the massive stars is neglected. This simplifying assumption will be discussed in the second part of $\S 4$, where a detailed energy balance between the amount of stellar energy lost to dust obscuration and the amount of FIR energy emitted by dust will be derived.

As mentioned in the Introduction, the decomposition of the FIR flux into two "effective temperature components" is a rough approximation of the reality, since there is probably a continuous distribution of temperatures for the dust. However, de Jong \& Brink (1987) and LH (see also Cox et al. 1986) have shown that the two-component approximation provides a simple but useful description of the characteristics of the emitting dust.

We have access only to two observables, $S 60$ and $S 100$, for the thermal emission from large dust grains, while the model has four free parameters, namely, the total emission FIR $=$ FIR $^{w}+$ FIR $^{c}$, the ratio FIR $^{w} / F^{\prime} R^{c}$, and the temperatures $T_{w}$ and $T_{c}$ of the warm and cool dust, respectively. Therefore, assumptions about some of the properties of the dust emission are required in order to evaluate the relative contributions of the cool and warm dust to the FIR emission. Our approach is to adopt values for both $T_{w}$ and $T_{c}$ and derive the other two parameters from the observed $S 60$ and $S 100$ fluxes. The only (weak) constraint $T_{w}$ and $T_{c}$ must obey is to have higher and lower values, respectively, of the mean dust temperature $T_{d}$ derived from the $S 60 / S 100$ ratios of the galaxies.

The galaxies in S1 are mostly late-type spirals or irregulars, so the cool dust emission can be assumed to have temperature $T_{c}=22 \mathrm{~K}$ (Buat \& Deharveng 1988). Taking a dust emissivity index $n=2$, the fraction of the cool infrared flux emitted in the range $40-120 \mu \mathrm{m}$ is $\operatorname{FIR}^{c}=0.53 F^{c}(1-1000 \mu \mathrm{m})$, and the ratio of the $60 \mu \mathrm{m}$ to the $100 \mu \mathrm{m}$ fluxes is: $S 60^{c} / S 100^{c}=0.17$. The small grain emission is not included in the derivation of the ratio $S 60^{c} / S 100^{c}$; this implies that the ratio is underestimated by about $20 \%$ (e.g., Désert et al. 1990), which is well within our uncertainties. Furthermore, because of the relatively hightemperature $T_{c}$ adopted for the cool dust emission, the ratio $S 60^{c} / S 100^{c}$ derived from thermal equilibrium calculations is not much different from the value 0.2 observed by Low et al. (1984) for Galactic cirrus and derived by Draine \& Anderson (1985; see also Désert 1986) when taking into account the emission from small grains.

The temperature of the warm dust component is in the range 30-50 K (Cox et al. 1986; Chini et al. 1986). We adopt $T_{w}=42$ $\mathrm{K}$, which is the rms of the dust temperatures of our sample galaxies derived from the color-corrected $S 60 / S 100$ ratios (Table II.A.1 in Fullmer \& Lonsdale 1989) in the hypothesis of a single-temperature component (see Table 2). In the case of starburst galaxies, where the warm component is the dominant contribution to the FIR emission, the adopted value of $T_{w}$ is a reasonable guess. $T_{w}=42 \mathrm{~K}$ yields $\mathrm{FIR}^{w}=0.78 F^{w}(1-1000$ $\mu \mathrm{m})$ and $S 60^{w} / S 100^{w}=1.27$. The emission from small grains has little effect on the values of $S 60^{w} / S 100^{w}$ and of FIR ${ }^{w}$.

By requiring $S 60=S 60^{c}+S 60^{w}$ and $S 100=S 100^{c}$ $+S 100^{w}$, we derive for each galaxy the relative fraction of the FIR emission due to FIR $^{c}$ and FIR ${ }^{w}$ (see Table 2). An example of decomposition of the FIR emission into the warm and cool components is given in Figure 3.

To impose the same two values $T_{w}$ and $T_{c}$ on all the galaxies might be too strong a constraint, because the two "effective temperatures" may differ from galaxy to galaxy. However, the $I R A S$ data do not allow us to constrain all four free parameters of the two-component model, and testing for variations in $T_{w}$ or $T_{c}$ is beyond the reach of the present data set.

From flux measurements at 100 and $155 \mu \mathrm{m}$, Hunter et al. (1989b) find that the infrared emission from NGC 1569 (one of the galaxies in S2) is compatible with a $100 \%$ warm dust emission at $T=34 \mathrm{~K}$. Our decomposition of the FIR flux from NGC 1569 attributes less than $10 \%$ of the emission to cool dust, in agreement with Hunter et al.'s result. Therefore, the two-component decomposition with constant temperatures provides reasonable answers even in the case of galaxies almost completely dominated by warm dust.

For the galaxies of the sample, Table 2 reports the blue luminosity derived from the RC3 magnitudes, the $\mathrm{H} \alpha$ luminosity, the electron temperature (see Storchi-Bergmann et al. 1994), the color excess $E_{l}(B-V)$ obtained from the ratio of the Balmer emission lines $\mathrm{H} \alpha / \mathrm{H} \beta$ (see CKS), the UV spectral index $\beta$, the FIR luminosity $L_{\mathrm{IR}}$ (eq. [7]), the ratio of the FIR luminosity to the blue luminosity, the temperature $T_{d}$ of the dust derived from the color-corrected $S 60 / S 100$ ratio (Young et al. 1989; see Table II.A.1 of Fullmer \& Lonsdale 1989 for the color-correction of the IRAS flux densities), the total mass of the dust, obtained assuming a single temperature dust component (Young et al. 1989), and the ratios FIR $^{w} /$ FIR and FIR $^{c} /$ FIR.

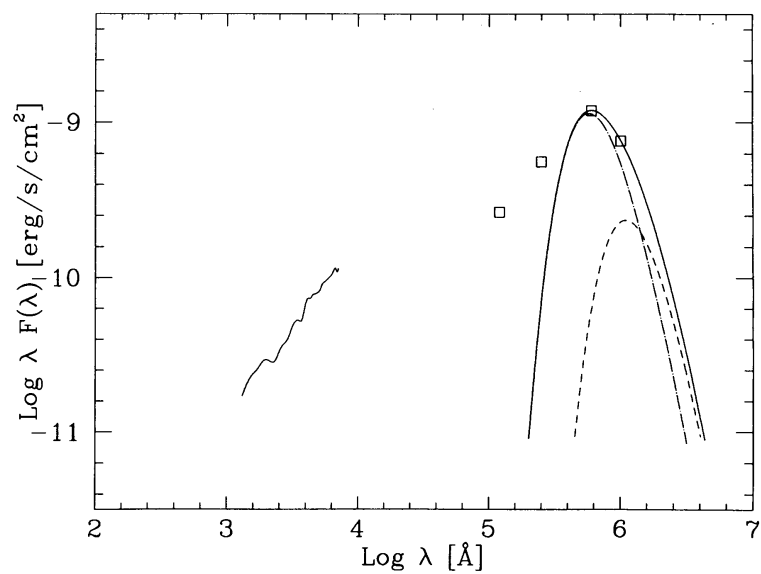

FIG. 3.-The UV, optical and far-infrared (infrared) spectral energy distribution, $\lambda F(\lambda)$ as a function of the wavelength for one of the galaxies in our sample, NGC 4194. The continuous line on the left-hand side of the diagram is the UV and optical spectral energy distribution; the squares are the infrared spectral energy distribution as obtained from the IRAS fluxes. On the same plot the two modified Planck curves, of the form $v^{2} B_{v}(T)$, representing the cool dust emission ( $T_{c}=22 \mathrm{~K}$, dashed line $)$ and the warm dust emission $\left(T_{w}=42 \mathrm{~K}\right.$, dot-dashed line), and the sum of the two curves (solid line) are shown. 
TABLE 2

DERIVED QUANTITIES For SAMPLE S1

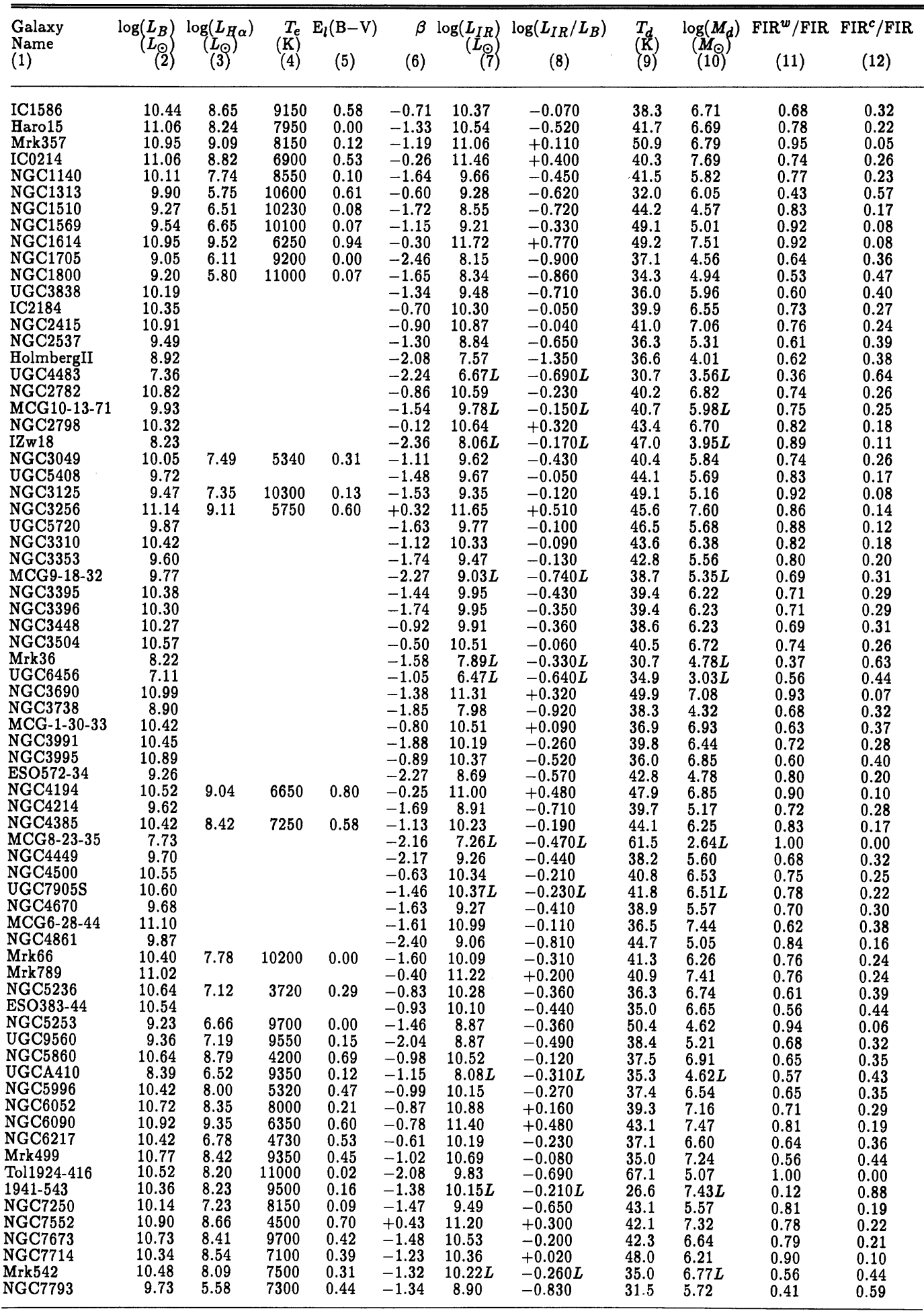

Notes.-The blue luminosity $L_{B}$ (col. [2]) is derived from the total blue magnitude of the galaxy (see Table 1). The $\mathrm{H} \alpha$ luminosity $L(\mathrm{H} \alpha)$ (col. [3]) is derived from the extinction-corrected $\mathrm{H} \alpha$ flux obtained from optical spectra of the central 200 square arcsec of the galaxy. The electron temperature $T_{e}$ (col. [4]), the color excess $E_{l}(B-V)$ obtained from the Balmer decrement (col. [5]), and the UV power-law index $\beta$ (col. [6]) have been derived by Calzetti et al. 1994 and by StorchiBergmann et al. 1994. The FIR luminosity $L_{\mathrm{IR}}$ (col. [7]) is given by the IRAS fluxes at 60 and $100 \mu \mathrm{m}$ through eq. (7). The dust temperature $T_{d}$ (col. [9]) and the dust mass $M_{d}$ (col. [10]) are derived from the $I R A S$ fluxes at 60 and $100 \mu \mathrm{m}$ in the hypothesis of a single temperature dust component (see Young et al. 1989). An L following a value in columns (7), (8), and (10) denotes un upper limit. FIR ${ }^{w} /$ FIR and FIR $/$ FIR (cols. [11] and [12]) are the fraction of FIR emission due to warm dust $\left(T_{w}=42 \mathrm{~K}\right.$ ) and cool dust $\left(T_{c}=22 \mathrm{~K}\right)$, respectively. 
The galaxies in our sample receive most of their FIR emission from the warm dust component, since there is ongoing star formation in their central regions. The rms values are $\left\langle\mathrm{FIR}^{w} / \mathrm{FIR}\right\rangle=0.70$ and $\left\langle\mathrm{FIR}^{c} / \mathrm{FIR}\right\rangle=0.30$. The FIR $^{c}$ fraction covers a relatively large range of values, going from $0 \%$ to more than $40 \%-50 \%$ in some of the galaxies.

\section{FROM ULTRAVIOLET OBSCURATION TO FAR-INFRARED EMISSION}

In this section, our goal is to understand how the dust obscuration estimated from the UV spectrum of the galaxy central region relates to the global properties of the galaxy, such as the FIR-to-blue ratio.

As will be shown, the amount of UV dust obscuration correlates with the "strength" of the FIR activity (the FIR-to-blue ratio); the correlation indicates that the stellar populations responsible for the observed UV and optical emission heat the dust which emits in the IRAS bands. Aperture mismatch between UV-optical and FIR data will be shown to be relatively unimportant for the present analysis.

\subsection{The Far-Infrared Properties}

The 72 starburst and blue compact galaxies in our sample have FIR luminosities in the range $5 \times 10^{7} \leq L_{\mathrm{IR}} \leq 5 \times 10^{11}$ and the FIR-to-blue ratio in the range $0.05 \leq L_{\mathrm{IR}} / L_{B} \leq 6.3$. Thus, these galaxies are relatively low-luminosity infrared emitters with a range of infrared-to-blue luminosity typical of normal galaxies (de Jong et al. 1984; Hunter et al. 1989a).

The IRAS color-color diagram is shown in Figure 4 and includes the galaxies with detections in at least three of the four passbands. Where necessary, an upper limit for the $12 \mu \mathrm{m}$ pass band has been used. The points are distributed along the sequence discussed by Helou (1986), mostly in the region where the warm dust component is dominant relative to the cool dust component, although some of the galaxies show colors which are compatible with $50 \%$ warm component and $50 \%$ cool component. Figure 4 confirms the result of the previous section, concerning the presence in some galaxies of an important contribution to the FIR emission from the cool dust heated by the general interstellar radiation field.

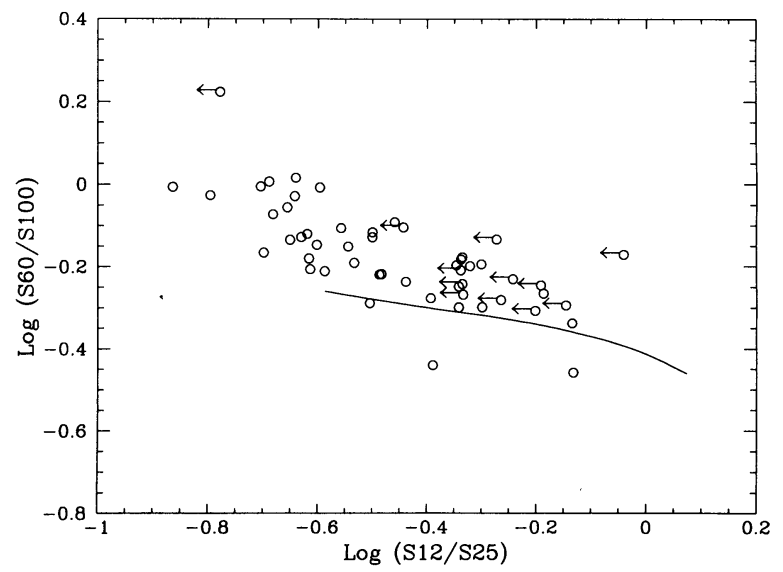

FIG. 4.-The IRAS color-color diagram for the 53 galaxies in our sample which either are detected in the four IRAS bands or have upper limits in the 12 $\mu \mathrm{m}$ band and are detected in the other three bands. The upper limits in the 12 $\mu \mathrm{m}$ band are indicated by the arrows. The continuous line is from Helou (1986) and marks the region where the $I R A S$ colors are consistent with $50 \%$ warm and $50 \%$ cool dust emission. The data are compatible with a mix of more than $50 \%$ warm dust and less than $50 \%$ cool dust.

\subsection{The Contributors to the Far-Infrared Emission}

Stellar radiation lost to dust absorption is the source of dust heating in nonactive galaxies. The following assumes that the ionizing radiation is associated with the warm dust FIR emission, and the nonionizing UV and optical radiation is entirely associated with the cool dust FIR emission. This rough assumption will be discussed at the end of $\$ 3.5$ and 4 .

The idea of identifying the GISRF heating the cool dust with the nonionizing UV spectrum from the galaxies (Boulanger \& Pérault 1988; Buat \& Deharveng 1988; Hunter et al. 1989a; $\mathrm{Xu}$, Lisenfeld, \& Völk 1994) is supported by the CKS results. CKS find a difference in the physical environment of $\mathrm{O}$ and early-B stars, which emit radiation predominantly below 912 $\AA$, and of intermediate-to-late B, A, and later type stars, which produce the UV and optical emission longward $912 \AA$. Hot ionizing stars are located in regions of higher dust content (a factor 2 on average) than colder nonionizing stars. On average, about $80 \%$ of the observed IUE flux $(1200-3200 \AA)$ is due to the less obscured B and A stars.

The FIR luminosity can be expressed as

$$
L_{\mathrm{IR}}=L_{\mathrm{IR}}^{c}+L_{\mathrm{IR}}^{w},
$$

where $L_{\mathrm{IR}}^{c}$ is the cool dust FIR luminosity and $L_{\mathrm{IR}}^{w}$ is the warm dust FIR luminosity. The first term on the right-hand side of equation (8) is given by (see, e.g., Belfort, Mochkovitch \& Dennefeld 1987):

$$
\begin{aligned}
L_{\mathrm{IR}}^{c} & =f^{c} L_{>912}\left[E_{c}(B-V)\right] \\
& =f^{c} \int_{912}^{8000} l(\lambda)\left[1-10^{-0.4 E_{c}(B-V) k(\lambda)}\right] d \lambda,
\end{aligned}
$$

where $l(\lambda)$ is the intrinsic luminosity of the galaxy at wavelength $\lambda$; the integrand is the difference between the intrinsic and the emergent spectral energy distributions (see eqs. [3][5]). The integral in equation (9) is the amount of UV and optical nonionizing radiation absorbed by dust and converted into infrared luminosity. The fraction of this radiation which contributes to the $40-120 \mu \mathrm{m}$ waveband is given by $f^{c}$. The obscuration curve $k(\lambda)$ is given by the sum of two processes: (1) absorption of radiation by dust and (2) scattering of radiation by dust off the line of sight. In principle, only the part of $k(\lambda)$ due to dust absorption contributes to the infrared emission in equation (9). However, in the following we assume that $k(\lambda)$ is mostly given by dust absorption, since in our sample the observational aperture is large enough to encompass most of the dust scattering regions.

In determining the fraction $f^{c}$ of nonionizing radiation contributing to the $40-120 \mu \mathrm{m}$ cool dust emission, two contributions must be considered: (1) the fraction $f_{\mathrm{lg}}^{c}$ of infrared energy emitted in the $40-120 \mu \mathrm{m}$ waveband by large grains at temperature $T_{c}\left(f_{\mathrm{lg}}^{c}=0.53\right.$, see $\left.\S 2.2\right)$; and (2) the fraction $f_{\mathrm{sg}}^{c}$ of nonionizing energy lost because of the heating of the large molecules and small grains (Xu 1990), which emit the bulk of their radiation at $\lambda<50 \mu \mathrm{m}$. Boulanger et al. (1988) and Puget \& Léger (1989) suggest that small grains/large molecules absorb about $40 \%-45 \%$ of the GISRF energy reemitted in the infrared. About three-quarters of this emission is at $\lambda<40 \mu \mathrm{m}$ (see Désert et al. 1990). Therefore, $f_{\mathrm{sg}}^{c} \simeq 0.30(\mathrm{Xu} 1990)$.

A more direct estimation of $f_{\text {sg }}^{c}$ can be obtained from the amount of infrared radiation emitted by our galaxies at $\lambda<40$ $\mu \mathrm{m}$. We assume that small grains are mainly heated by the nonionizing radiation, since the ionizing radiation is energetic enough to destroy them (e.g., Boulanger et al. 1988; Telesco, 
Decher, \& Joy 1989). In sample S1, 41 galaxies have detections at 12 and $25 \mu \mathrm{m}$. For these galaxies, the infrared luminosity in the range 10-40 $\mu \mathrm{m}$ (Rowan-Robinson \& Crawford 1989) is, on average, $L_{\mathrm{IR}}^{\text {obs }}(10-40 \mu \mathrm{m}) \simeq 0.40 L_{\mathrm{IR}}$. From this value we must subtract the contribution to the $10-40 \mu \mathrm{m}$ band of the large grains in thermal equilibrium at $T=T_{c}$ and $T=T_{w}$, which is $L_{\mathrm{IR}}^{\mathrm{lg}}(10-40 \mu \mathrm{m}) \simeq 0.13 L_{\mathrm{IR}}$, for values of $\mathrm{FIR}^{c} / \mathrm{FIR}^{w}$ typical of our galaxies. The excess of the observed $L_{\mathrm{IR}}(10-40$ $\mu \mathrm{m})$ relative to the calculated one, $L_{\mathrm{IR}}^{\mathrm{obs}}-L_{\mathrm{IR}}^{\mathrm{lg}} \sim 0.25$, can be attributed to emission from small grains. Therefore, $f_{\mathrm{sg}}^{c} \simeq 0.25$, a value in agreement with the previous estimation, considering that the infrared emission below $10 \mu \mathrm{m}$ is not included in the present derivation.

In summary, the fraction $f^{c}$ of nonionizing radiation contributing to the $40-120 \mu \mathrm{m}$ cool dust emission is $f^{c}=$ $f_{\mathrm{lg}}^{c}\left(1-f_{\text {sg }}^{c}\right)=0.37$.

The FIR luminosity due to emission from warm dust can be expressed as

$$
\begin{aligned}
L_{\mathrm{IR}}^{w} & =f^{w} L_{\mathrm{ion}} \\
& =f^{w}\left[f N_{\mathrm{Lyc}} h v_{\mathrm{Ly} \alpha}+(1-f) N_{\mathrm{Lyc}}\left\langle h v_{\mathrm{Lyc}}\right\rangle\right],
\end{aligned}
$$

where $L_{\text {ion }}$ is the luminosity of the ionizing photons which is absorbed by dust; $f^{w}$ is the fraction of infrared energy emitted in the waveband $40-120 \mu \mathrm{m}$ by the large grains in thermal equilibrium at $T=T_{w}\left(f^{w}=0.78\right) ; N_{\mathrm{Lyc}}$ is the number of ionizing photons per second; and $(1-f)$ is the fraction absorbed directly by dust, while $f$ is the fraction of ionizing photons processed by the gas, so that only the resulting Ly $\alpha$ photons are absorbed by dust. We have adopted the simplifying assumption that each Lyman continuum photon absorbed by the gas produces a Ly $\alpha$ photon (see, however, Mezger, Mathis, \& Panagia 1982); this assumption introduces an overestimation of $L_{\text {ion }}$ of the order of $20 \%-25 \%$, well within our typical uncertainties. The first term on the right-hand side of equation (10) takes into account the fact that a fraction of the ionizing flux emerges in the nebular lines. The number of ionizing photons per second can be derived from the $\mathrm{H} \alpha$ emission through the following relation (Lequeux 1980; Belfort et al. 1987):

$$
N_{\mathrm{Lyc}}=7.35 \times 10^{11} \frac{1}{f} L(\mathrm{H} \alpha)\left(\frac{T_{e}}{10^{4}}\right)^{-0.09},
$$

where $L(\mathrm{H} \alpha)$ and $T_{e}$, the electron temperature, are given in Table 2. In our calculations, the ionizing flux per unit wavelength, $F_{\text {ion }}(\lambda)$, is assumed constant in the range 300-912 $\AA$ and is zero below $300 \AA$, which implies $\left\langle\lambda_{\text {Lyc }}\right\rangle \simeq 0.5 \lambda_{\text {Ly } \alpha}$ (see Mezger 1978). In addition, the fraction of ionizing photons directly absorbed by dust is assumed to be $(1-f) \simeq 0.35$ (Smith, Biermann, \& Mezger 1978; Mezger 1978).

The FIR luminosity is normalized to the observed blue luminosity of the galaxy $\left(L_{\mathrm{IR}} / L_{B}\right)$ to account for variations in size and/or mass of the galaxies in the sample and for possible distance-dependent selection effects. The observed blue luminosity is given by

$$
L_{B}=L_{B}^{i} 10^{-0.4 E_{c}(B-V) k_{B}},
$$

where $L_{B}^{i}$ is the intrinsic blue luminosity of the galaxy. Equation (12) assumes that the blue luminosity of the entire galaxy is subject to an obscuration given by the color excess $E_{c}(B-V)$, derived from the galaxy central region. This is an approximation of the average color excess in the galaxy, since the central regions are typically more extincted than, or at least as much extincted as, the outer regions (e.g., van der Hulst et al. 1988; Belley \& Roy 1992; Martin \& Roy 1992). We will use equation (12) to derive $L_{B}^{i}$ only for sample $S 2$, where most of the blue light comes from the galaxy central regions, and $E_{c}(B-V)$ represents a reasonable value for the color excess.

\subsection{The Correlation between the Ultraviolet Obscuration and the Far-Infrared Emission}

As discussed in $\S 2.1$, the UV spectral index $\beta$ can be used as a measure of the effective dust obscuration affecting the emerging UV spectrum in the wavelength range $1200-2600 \AA$.

Figure 5 shows $\beta$ plotted as a function of the FIR-to-blue luminosity, $L_{\mathrm{IR}} / L_{B}$. The two quantities, $\beta$ and $\log \left(L_{\mathrm{IR}} / L_{B}\right)$ are clearly correlated: a Spearman rank correlation test shows that the $\beta$ versus $\log \left(L_{\mathrm{IR}} / L_{B}\right)$ relation deviates $5.6 \sigma$ from the null hypothesis (5.3 $\sigma$, if NGC 1313 is included). A linear fit gives

$$
\beta=(1.12 \pm 0.13) \log \left(L_{\mathrm{IR}} / L_{B}\right)-(0.94 \pm 0.06) .
$$

The theoretical value $\beta(0)=-2.3$ for a (almost) dust-free burst of star formation is given by $L_{\mathrm{IR}} / L_{B}=0.06$, the smallest ratio in our sample.

The correlation between $\beta$ and the ratio of the dust mass to the blue luminosity $\left(M_{d} / L_{B}\right)$ deviates $5.7 \sigma$ from the null hypothesis (6.0 $\sigma$ if the upper limits are excluded, see Fig. 6). $M_{d}$ is the warm dust mass determined in the hypothesis of a singletemperature component (Young et al. 1989; see cols. [9] and [10] of Table 2); within the single-component model the uncertainties on the warm dust mass are $\sim 30 \%$. However, the warm dust mass is probably a small fraction of the total dust mass: within the two-component model presented here, the rms cool dust/warm dust mass ratio for the galaxies in $\mathrm{S} 1$ is about 15 . This value is likely to be underestimated, because cold dust at temperatures $T \lesssim 15 \mathrm{~K}$ is not detected by $I R A S$ (e.g., Kwan \& Xie 1992; Draine 1990; see, however, Thronson et al. 1990).

Figures 5 and 6 also indicate that S2 (shown with filled symbols) is a representative subsample of $\mathrm{S} 1$. Correlations between $\beta$ and $L_{\mathrm{IR}} / L_{B}$ and between $\beta$ and $M_{d} / L_{B}$ are evident for S2: the Spearman rank correlation gives deviations of $4.6 \sigma$

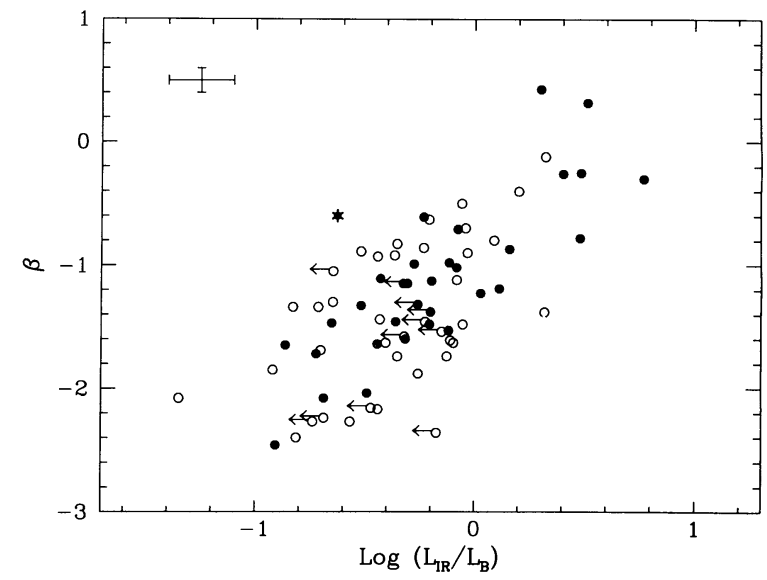

FIG. 5.-The UV spectral index $\beta$, a dust obscuration indicator, as a function of $\log \left(L_{\mathrm{IR}} / L_{B}\right)$, which measures the "strength" of the FIR activity of the galaxy. The data are relative to sample S1. The filled dots are the galaxies in common between sample S1 and sample S2. The star shows the position of NGC 1313. $L_{B}$ is the total blue luminosity of the galaxy, derived from the RC3 $B_{T}^{0}$ or other integrated blue luminosities. Upper limits on the FIR luminosity $L_{\mathrm{IR}}$ are indicated by arrows. In the upper left-hand corner of the figure the typical $1 \sigma$ error bar is shown. Typical uncertainties are $20 \%$ on $L_{\mathrm{IR}}$ and 0.2 mag on $L_{B}$. 


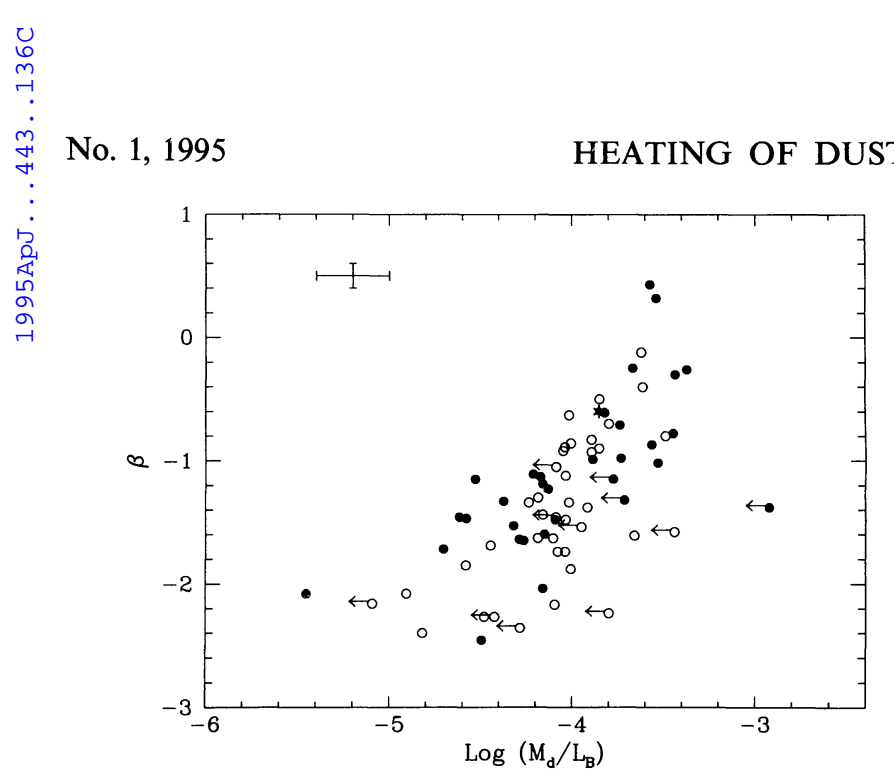

FIG. 6.-The UV spectral index $\beta$ as a function of $\log \left(M_{d} / L_{B}\right)$, which measures the specific amount of dust per unit of blue luminosity in the galaxy. Symbols are as in Fig. 5.

and $4.3 \sigma$, respectively, from the null hypothesis. In Figure 7, the intrinsic excess $E_{l}(B-V)$ of the galaxies is shown as a function of $L_{\mathrm{IR}} / L_{B}$; as expected, the two quantities are correlated at $4.5 \sigma$ from the null hypothesis. In fact, both $\beta$ and $E_{l}(B-V)$ are indicators of dust obscuration, though $\beta$ has a larger dynamical range than $E_{l}(B-V)$.

In principle, the interpretation of Figures 5 and 6 is straightforward: an increase of the amount of dust in the galaxy corresponds to a lower blue luminosity (larger obscuration) and to a larger infrared emission, implying increasing ratios $L_{\mathrm{IR}} / L_{B}$ and $M_{d} / L_{B}$. However, for this to be true, we have to exclude biases or selection effects in the data. The next section is devoted to this task.

\subsection{Selection Effects in the $\beta-$ Far-Infrared Correlation}

There are two different selection effects our sample must be tested for: (1) IUE-optical and IRAS apertures mismatch: (2) sensitivity limits of the two instruments, IUE and IRAS.

Effects of the aperture mismatch between the UV and infrared data can be investigated by studying how galaxies of differ-

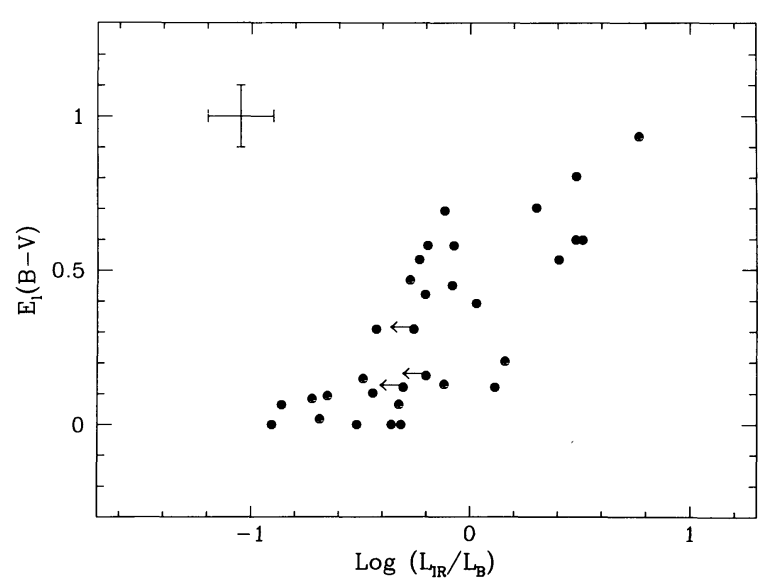

Fig. 7.-The color excess $E_{l}(B-V)$, determined from the Balmer decrement, as a function of the ratio $\log \left(L_{\mathrm{IR}} / L_{B}\right)$. The data are relative to sample $\mathrm{S} 2$, the galaxies with optical spectra. The increasing trend of the FIR-to-blue ratio in the galaxies for increasing values of the dust obscuration can be seen from this diagram.

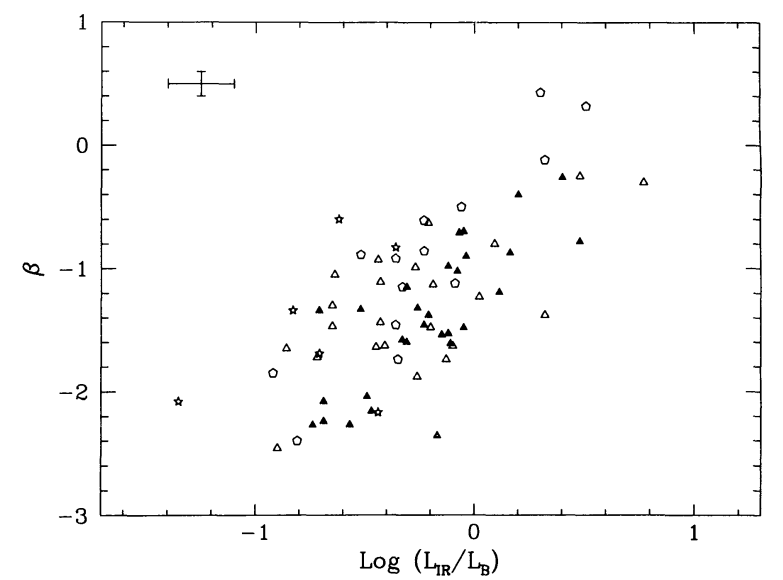

FIG. 8.-The plot of Fig. 5 is shown again, attributing different symbols to galaxies with apparent diameter $d<1^{\prime}$ (filled triangles), $1^{\prime} \leq d<2^{\prime}$ (open triangles), $2^{\prime} \leq d<5^{\prime}$ (open pentagons), and $d \geq 5^{\prime}$ (stars).

ent apparent size distribute in the plane $\beta$ - $\log \left(L_{\mathrm{IR}} / L_{B}\right)$. Figure 8 reports the same data of Figure 5 with different symbols ascribed to different ranges of the galaxy angular diameter $d$. The angular diameter is defined as the rms of the minor and major apparent diameters of the galaxy. Galaxies of different angular diameter scatter along the sequence $\beta-\log \left(L_{\mathrm{IR}} / L_{B}\right)$, without crowding in any particular region of the diagram. Therefore, the $\beta$ versus $L_{\mathrm{IR}} / L_{B}$ correlation is not a product of the aperture mismatch.

Figure 8 also shows that the largest galaxies $\left(d<5^{\prime}\right)$ tend to populate the upper side of the relation $\beta$ versus $L_{\mathrm{IR}} / L_{B}$. Such behavior can be understood if a large fraction of the FIR emission is generated in a smaller region than the blue luminosity, in which case larger galaxies do not necessarily have more extended FIR emission. For example, In NGC 5253 most of the infrared emission at $12 \mu \mathrm{m}$ originates within the central 60 pc, namely, $4^{\prime \prime}$, in a region coinciding with the central burst of star formation (Telesco, Dressler, \& Wolstencroft 1993). The trend of the infrared emission to be centrally concentrated in actively star-forming galaxies is confirmed in sample S2 (Fig. 5 ), where extended galaxies have been removed and the correlation $\beta$ versus $L_{\mathrm{IR}} / L_{B}$ shows a smaller dispersion than in the entire sample $\mathrm{S} 1$.

Since the detection of the UV spectrum has been the selection criterion for our sample, sensitivity limits of the UV instrument can produce selection effects on the sample. Both high dust obscuration and low SFRs contribute to give low UV fluxes, thus removing objects from the UV sample. In addition, dust obscuration and SFR compete in producing the ratio $L_{\mathrm{IR}} / L_{B}$. An increase of the dust obscuration decreases $L_{B}$ and increases the FIR emission, because a larger fraction of nonionizing stellar radiation heats the dust. A decrease in the SFR decreases $L_{B}$ and the FIR emission due to warm dust, because a smaller number of massive stars is available to heat the dust. An analysis of the diagram $\beta$ versus $\log \left(L_{\mathrm{IR}} / L_{B}\right)$ in Figure 5 can shed light on this issue. The bottom right-hand corner of the diagram is unlikely to be affected by selection effects: this region corresponds to small values of $\beta$, namely, unobscured galaxies, and large values of $L_{\mathrm{IR}} / L_{B}$, which means large SFRs. Galaxies with such properties would be detectable by both the UV and infrared instruments, because they would have both strong UV fluxes and large FIR fluxes. The fact that the bottom right-hand corner of Figure 5 is not populated 
implies that it is unlikely to have galaxies unobscured at UV wavelengths and with a strong infrared emission.

The region of the $\beta$ versus $\log \left(L_{\mathrm{IR}} / L_{B}\right)$ diagram corresponding to large values of $\beta$ with small $L_{\mathrm{IR}} / L_{B}$ ratios, namely, the upper left-hand corner of Figure 5, is the region which may be artificially depleted because of high obscuration and low SFRs. Such is the case of metal-rich, dusty galaxies with small present SFRs, and with a large late-A and $F$ stellar population (contributing to the blue luminosity). This selection effect cannot be excluded from the present sample; however, it does not affect the results of the previous sections as long as the correlation $\beta$ versus $\log \left(L_{\mathrm{IR}} / L_{B}\right)$ corresponds to a sequence of increasing dust content and not to a sequence of increasing SFR.

The sample $\mathrm{S} 2$ can be used to investigate whether the increase in dust content or the increase in SFR is dominant in producing the correlation $\beta$ versus $\log \left(L_{\mathrm{IR}} / L_{B}\right)$.

The ratio between the luminosity of the ionizing photons and the intrinsic blue luminosity $L_{\text {ion }} / L_{B}^{i}$ gives a measure of the SFR per solar blue luminosity, and, hence, a measure of the current relative SFR (Kennicutt 1983). Another measure of the relative SFR is the quantity $L_{\text {ion }} /$ area, which is the rate of ionizing photons weighted for the physical size of the observed galaxy region (Hunter et al. 1989a). The luminosities $L_{\text {ion }}$ have been calculated from equations (10)-(11), using the values of the $\mathrm{H} \alpha$ luminosity and the electron temperature reported in Table 2. The $\mathrm{H} \alpha$ fluxes have been extinction corrected using the color excesses $E_{l}(B-V)$ listed in Table 1 and a Seaton extinction law. The bluc luminosities $L_{B}^{i}$ are derived from the optical spectra, after the stellar continua have been obscuration-corrected with the recipe given in $\S 2.1$, equations (3)-(5).

In Figure $9 a, L_{\text {ion }} / L_{B}^{i}$ is plotted as a function of $L_{\mathrm{IR}} / L_{B}$. There is no trend of the relative SFR with the FIR-to-blue luminosity ratio, indicating that the SFR is not the discriminating parameter which determines the sequence $\beta$ versus $L_{\mathrm{IR}} / L_{B}$. In Figure $9 b$, the ratio $L_{\text {ion }} /$ area is shown as a function of $L_{\mathrm{IR}} / L_{B}$. The correlation is only $2.7 \sigma$ from the null hypothesis, and thus is too weak to cause the $\beta$ versus $L_{\mathrm{IR}} / L_{B}$ correlation.

The conclusion of this section is that the sequence $\beta$ versus $\log \left(L_{\mathrm{IR}} / L_{B}\right)$ is not affected by selection effects in the sample and is not due to increasing relative SFR in more obscured galaxies. The main cause of the sequence appears to be a progressively higher dust content in galaxies with larger FIR-toblue ratios.

\subsection{The Meaning of the $\beta$-versus-Far-Infrared Correlation}

The optical data available for the galaxies in S2 allow further investigation of the correlation between $\beta$ and $L_{\mathrm{IR}} / L_{B}$.

The variations of the relative SFR in the $\beta$ versus $\log$ $\left(L_{\mathrm{IR}} / L_{B}\right)$ plot are shown in Figure 10 , where different symbols are attributed to the data points according to their value of the ratio $L_{\text {ion }} / L_{B}^{i}$. Despite the scatter in the diagram, there is the indication of a trend: for constant values of $\beta$, galaxies with higher SFR have higher ratios $L_{\mathrm{IR}} / L_{B}$. This trend is expected, since for constant dust content, an increase in the SFR produces an increase in the FIR emission. Thus, the best-fit line,

$$
\beta=(1.29 \pm 0.16) L_{\mathrm{IR}} / L_{B}-(0.91 \pm 0.07)
$$

locates points of increasing dust content but constant $L_{\text {ion }} / L_{B}^{i}$. The trend can probably explain why previous authors (e.g., Salzer \& MacAlpine 1988) do not find a correlation between the intrinsic color excess and the FIR-to-blue ratio of galaxies.
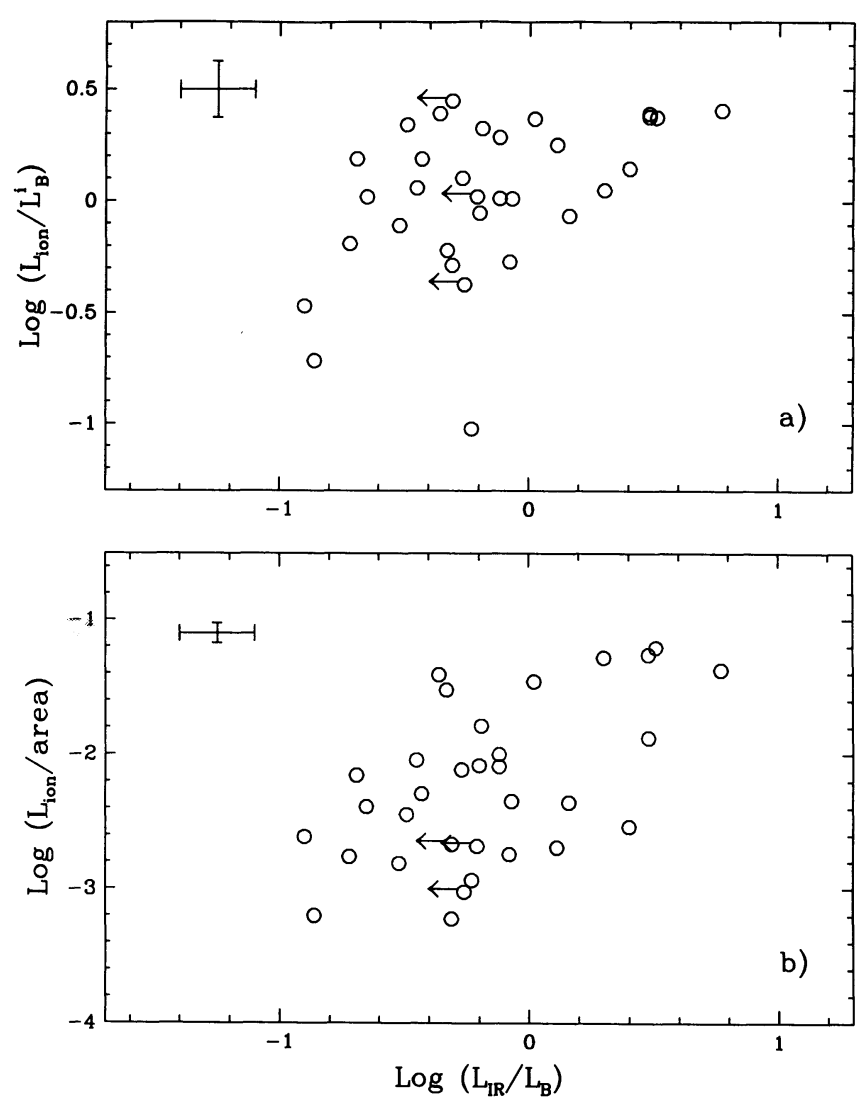

FIG. 9. $-(a)$ The current relative star formation rate (SFR), $\log \left(L_{\text {ion }} / L_{B}^{i}\right)$, as a function of the FIR activity, $\log \left(L_{\mathrm{IR}} / L_{B}\right)$, for the 32 galaxies in sample $S 2$. Arrows indicate upper limits on $L_{\mathrm{IR}} / L_{B}$. Both $L_{\text {ion }}$ and $L_{B}^{i}$ are derived from the optical spectra, after reddening correction. $(b)$ The rate of ionizing photons weighted by the size of the observed region, $\log \left(L_{\text {ion }} /\right.$ area $)$, as a function of $\log \left(L_{\mathrm{IR}} / L_{B}\right)$, The $\log \left(L_{\mathrm{ion}} /\right.$ area $)$ is a measure of the current SFR per unit area.

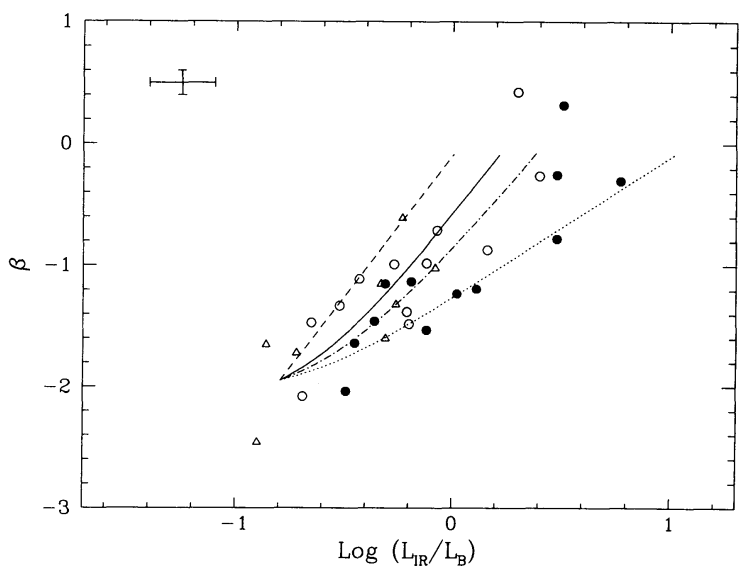

FIG. 10.-Different symbols are ascribed to different levels of the current relative SFR in this plot of $\beta$-versus-log $\left(L_{\mathrm{IR}} / L_{B}\right)$. Filled circles indicate $\log$ $\left(L_{\text {ion }} / L_{B}^{i}\right)>0.80$, open circles indicate $0.80 \geq \log \left(L_{\text {ion }} / L_{B}^{i}\right)>-0.15$, and triangles indicate $\log \left(L_{\text {ion }} / L_{B}^{i}\right) \leq-0.15$. The four lines represent the models described in $\S 3.5$ : (1) constant relative SFR and contribution to $L_{\mathrm{IR}}$ from the ionizing radiation alone (dashed line); (2) constant relative SFR and contribution to $L_{\mathrm{IR}}$ from both the ionizing and the nonionizing radiation (solid line); (3) same as case 2, but adopting $E_{c}(B-V)=E_{l}(B-V)$ (dotted line); (4) same as case 2 , but including the contribution to the warm dust emission from the nonionizing radiation of the massive stars (dot-dashed line). 
The correlation between dust obscuration and dust emission can be hidden by a substantial variation in the SFR values of the galaxies considered. In addition, previous works have made use of the intrinsic color excess $E_{l}(B-V)$ to quantify optical dust obscuration; however, $E_{l}(B-V)$ has a smaller dynamical range than the UV power-law index $\beta$, thus increasing the difficulty of defining correlations between UV-optical and FIR data.

The correlation $\beta$ versus $\log \left(L_{\mathrm{IR}} / L_{B}\right)$ can be quantified by equations (8)-(12), writing

$$
\begin{aligned}
\frac{L_{\mathrm{IR}}}{L_{B}}= & \frac{f^{w} L_{\text {ion }}}{L_{B}^{i}} 10^{0.4 E_{c}(B-V) k_{B}} \\
& +\frac{f^{c} L_{>912}\left[E_{c}(B-V)\right]}{L_{B}^{i}} 10^{0.4 E_{c}(B-V) k_{B}} .
\end{aligned}
$$

Each term in equation (15) can be estimated by adopting the typical values for the ratio $\mathrm{FIR}^{w} / \mathrm{FIR}^{c}=f^{w} L_{\text {ion }} / f^{c} L_{>912}$ $\left[E_{c}(B-V)\right] \simeq 2.3$ shown by our data, and by imposing that for $E_{c}(B-V)=0$ the theoretical $L_{\mathrm{IR}} / L_{B}$ reproduces the smallest observed ratio $L_{\mathrm{IR}} / L_{B}$ reported in Figure 10. Three curves derived from equation (15) are shown in Figure 10: the first (dashed line) is the FIR-to-blue ratio as given by the first term on the right-hand side on equation (15), which is the contribution from the ionizing radiation alone. Since $L_{\text {ion }} / L_{B}^{i} \sim$ const, the variation of $L_{\mathrm{IR}} / L_{B}$ is produced by the loss of blue luminosity due to dust obscuration. The second curve (solid line) is the FIR-to-blue ratio as given by both the terms on the righthand side of equation (15). The agreement with the data points has certainly improved relative to the first curve, meaning that the contribution to the FIR emission from nonionizing radiation is important for reproducing the observed trend. For comparison, a third curve is shown (dotted line), obtained again from equation (15), with the hypothesis $E_{c}(B-V)=E_{l}(B-V)$. The third model is not steep enough to agree with the data, though none of the three curves gives a satisfactory fit. We attribute the disagreement to the simplifying assumption of neglecting the non-ionizing contribution of the massive stars to the warm dust emission. We can relax this constraint, and assume that the nonionizing flux associated with the massive stars is about 3 times the ionizing flux and is affected by the same dust obscuration as the ionizing radiation $\left(E_{l}[B-V]\right)$. We also assume that the entire radiation from the massive stars heats the dust which produces the warm FIR emission. The resulting curve on the $\beta$ versus $\log \left(L_{\mathrm{IR}} / L_{B}\right)$ plane is shown in Figure 10 (dot-dashed line). The agreement with the data points has improved relative to the previous three curves.

\section{THE ENERGY BALANCE}

This section is centered on the investigation of the energy balance between the UV-optical radiation and the FIR emission. The amount of stellar energy absorbed by dust at UVoptical wavelengths is estimated and compared with the amount of energy emitted by the dust in the infrared for the galaxies in $\mathrm{S} 2$.

The infrared emission probes deeper regions in a galaxy than UV or optical emission, since the infrared is subject to less extinction. UV and optical spectra of galaxies contain information on the fraction of a galaxy's stellar populations which is unobscured or mildly obscured. Heavily extincted stars do not contribute UV or optical flux. The infrared emission from dust provides information on the entire stellar population responsible for the heating. A detailed evaluation of the difference between the amount of energy absorbed by dust at UV and optical wavelengths and the amount of energy reemitted at infrared wavelengths provides insight into the amount of " hidden stars" in a galaxy.

The obscuration curve described in $\S 2.1$ and the values for the color excess $E_{c}(B-V)$ listed in Table 3 are used to deredden the UV-optical spectra in S2. The difference between the obscuration-corrected and the emergent spectra, $\delta F_{(912-8000)}=F_{(912-8000)}^{i}-F_{(912-8000)}^{\text {obs }}$, gives the amount of energy lost to dust obscuration; a fraction $f^{c}$ of this energy gives a prediction on FIR $^{c}$ (see Fig. 2). As mentioned in $\S 3.2$, the coincidence between dust obscuration and dust absorption is assumed, since most of the dust scattering regions are included in the observational aperture.

The procedure to evaluate $\delta F_{(912-8000)}$ is the following. Both emergent and obscuration-corrected spectra are fitted at UV wavelengths with power laws of indices $\beta$ and $\beta_{i}$, respectively (see eq. [1] and Table 3 ). The spectra cover the range $1200-8000 \AA$ (or, in some cases, $1200-2000 \AA$ and $3200-8000$ $\AA$ ). The amount of energy in the range $912-1200 \AA$ is estimated by extrapolating the power laws obtained from the fits at longer wavelengths. The same extrapolation is applied to the spectra of the galaxies lacking the wavelength range 2000-3200 $\AA$. The difference between the integrals of the obscurationcorrected and the emergent spectra in the range 912-8000 $\AA$ gives the amount of energy lost to dust obscuration.

The ionizing flux, $F_{\text {ion }}$, is estimated as in equations (10) and (11) from the extinction-corrected $\mathrm{H} \alpha$ flux (see $\S 3.4$ ). The fraction $f^{w}$ of $F_{\text {ion }}$ which has been converted into warm dust emission gives a prediction on FIR ${ }^{w}$.

Table 3 lists the galaxies of sample $S 2$, the fraction of blue light encircled by the $I U E$-optical aperture, the intrinsic color excess of the stellar continua $E_{c}(B-V)$, the observed and the dereddened UV spectral indices, $\beta$ and $\beta_{i}$, the total ionizing flux $F_{\text {ion }}$, the total flux in the range $912-8000 \AA$ of the emergent spectra $F_{(912-8000)}^{\text {obs }}$, the total flux in the range $912-8000 \AA$ of the obscuration-corrected spectra $F_{(912-8000)}^{i}$, the ratio between the predicted and observed warm dust emission $f^{w} F_{\text {ion }} /$ FIR $^{w}$, and ratio between the predicted and observed cool dust emission $f^{c} \delta F_{(912-8000)} /$ FIR $^{c}$.

Four galaxies in our sample, NGC 1705, NGC 5253, Haro 15 , and Mrk 66, have formally zero obscuration, $E_{l}(B-V)=$ $0.0 \pm 0.1$. In order to constrain the maximum amount of UV and optical radiation lost to dust obscuration, we have adopted for the four galaxies the maximum value for the obscuration allowed by the uncertainty, which means $E_{l}(B-V)=0.1$ and $E_{c}(B-V)=0.5 \times E_{l}(B-V)=0.05$ (see the values in parentheses and the footnote in Table 3$)$.

The predicted-to-observed warm dust emission, $f^{w} F_{\text {ion }} /$ FIR $^{w}$, and the predicted-to-observed cool dust emission, $f^{c} \delta F_{(912-8000)} /$ FIR $^{c}$, (cols. [9] and [10] of Table 3) are shown as a function of $L_{\mathrm{IR}} / L_{B}$ in Figures $11 a$ and $11 b$, respectively. The ionizing radiation accounts for $20 \%$, on average, of the warm FIR emission, and this contribution is independent of the ratio $L_{\mathrm{IR}} / L_{B}$. The spread of the data around the median value is about $1.2 \mathrm{dex}$, ranging from $f^{w} F_{\text {ion }} / \mathrm{FIR}^{w} \simeq 0.06$ to $f^{w} F_{\text {ion }} / \mathrm{FIR}^{w} \simeq 0.90$. The predicted-to-observed cool dust emission $f^{c} \delta F_{(912-8000)} /$ FIR $^{c}$ has median value 1 , independently of $L_{\mathrm{IR}} / L_{B}$; it implies that the observed loss of UV and optical radiation due to dust obscuration entirely accounts for the cool dust emission. The spread around the median value of $f^{c} \delta F_{(912-8000)} /$ FIR $^{c}$ is about 1.2 dex, covering the range $0.25-$ 
TABLE 3

DERIVED QuANTITIES FOR SAMPLE S2

\begin{tabular}{|c|c|c|c|c|c|c|c|c|c|}
\hline $\begin{array}{l}\text { Galaxy } \\
\text { Name } \\
(1)\end{array}$ & $\begin{array}{c}\left(L_{B}^{I U E} / L_{B}\right) \\
(2)\end{array}$ & $\begin{array}{l}E_{c}(B-V) \\
(3)\end{array}$ & (4) & $\begin{array}{l}\beta_{i} \\
(5)\end{array}$ & $\begin{array}{l}F_{i o n} \\
\mathrm{erg} / \mathrm{s} / \mathrm{cm}^{2} \\
(6)\end{array}$ & $\begin{array}{l}F_{(912-8000)}^{o b s} \\
\mathrm{erg} / \mathrm{s} / \mathrm{cm}^{2} \\
(7)\end{array}$ & $\begin{array}{l}F_{(912-8000)}^{i} \\
\mathrm{erg} / \mathrm{s} / \mathrm{cm}^{2} \\
(8)\end{array}$ & $\begin{array}{l}f^{w} F_{i o n} / F I R^{w} \\
(9)\end{array}$ & $\begin{array}{l}f^{c} \delta F_{(912-8000)} / F I R^{c} \\
(10)\end{array}$ \\
\hline IC1586 & 0.90 & 0.29 & -0.71 & -2.07 & $2.36 \mathrm{E}-11$ & $2.99 \mathrm{E}-11$ & $1.65 \mathrm{E}-10$ & 0.52 & 2.97 \\
\hline Haro15 & 0.35 & $0.00(0.05)$ & -1.33 & -1.33 & $8.15 \mathrm{E}-12(1.02 \mathrm{E}-11)$ & $6.75 \mathrm{E}-11$ & $6.75 \mathrm{E}-11(9.56 \mathrm{E}-11)$ & $0.12(0.15)$ & $0.00(0.67)$ \\
\hline Mrk357 & 1.03 & 0.06 & -1.19 & -1.74 & $1.08 \mathrm{E}-11$ & $4.17 \mathrm{E}-11$ & $6.01 \mathrm{E}-11$ & 0.21 & 3.10 \\
\hline NGC1140 & 0.65 & 0.05 & -1.64 & -1.85 & $4.76 \mathrm{E}-11$ & $1.96 \mathrm{E}-10$ & $2.60 \mathrm{E}-10$ & 0.28 & 0.60 \\
\hline NGC1510 & 0.41 & 0.04 & -1.72 & -1.80 & $9.13 \mathrm{E}-12$ & $7.15 \mathrm{E}-11$ & $9.09 \mathrm{E}-11$ & 0.20 & 0.99 \\
\hline NGC1569 & 0.34 & 0.03 & -1.15 & -1.31 & $1.59 \mathrm{E}-10$ & $1.38 \mathrm{E}-09$ & $1.70 \mathrm{E}-09$ & 0.05 & 0.64 \\
\hline NGC1614 & 0.72 & 0.47 & -0.30 & -2.57 & $2.24 \mathrm{E}-10$ & $8.56 \mathrm{E}-11$ & $8.45 \mathrm{E}-10$ & 0.13 & 2.52 \\
\hline NGC1705 & 0.61 & $0.00(0.01)$ & -2.46 & -2.46 & $1.28 \mathrm{E}-11(1.34 \mathrm{E}-11)$ & $4.89 \mathrm{E}-10$ & $4.89 \mathrm{E}-10(5.36 \mathrm{E}-10)$ & $0.25(0.26)$ & $0.00(0.81)$ \\
\hline NGC1800 & 0.33 & 0.03 & -1.65 & -1.81 & 3.32E-12 & $7.26 \mathrm{E}-11$ & $8.66 \mathrm{E}-11$ & 0.10 & 0.22 \\
\hline NGC3049 & 0.21 & 0.15 & -1.11 & -1.75 & $2.70 \mathrm{E}-11$ & $5.81 \mathrm{E}-11$ & $1.40 \mathrm{E}-10$ & 0.19 & 0.80 \\
\hline NGC3125 & 0.60 & 0.07 & -1.53 & -1.81 & $5.27 \mathrm{E}-11$ & $1.41 \mathrm{E}-10$ & $2.08 \mathrm{E}-10$ & 0.20 & 1.38 \\
\hline NGC3256 & 0.27 & 0.30 & +0.32 & -1.07 & $3.29 \mathrm{E}-10$ & $2.34 \mathrm{E}-10$ & $1.08 \mathrm{E}-09$ & 0.06 & 0.49 \\
\hline NGC4385 & 0.36 & 0.29 & -1.13 & -2.36 & $8.58 \mathrm{E}-11$ & $9.40 \mathrm{E}-11$ & $4.82 \mathrm{E}-10$ & 0.35 & 3.68 \\
\hline Mrk66 & 0.90 & $0.00(0.05)$ & -1.60 & -1.60 & $3.17 \mathrm{E}-12(3.98 \mathrm{E}-12)$ & $2.88 \mathrm{E}-11$ & $2.88 \mathrm{E}-11(4.08 \mathrm{E}-11)$ & $0.12(0.15)$ & $0.00(0.69)$ \\
\hline NGC5253 & 0.28 & $0.00(0.05)$ & -1.46 & -1.46 & $2.07 \mathrm{E}-10(2.60 \mathrm{E}-10)$ & $5.85 \mathrm{E}-10$ & $5.85 \mathrm{E}-10(8.22 \mathrm{E}-10)$ & $0.12(0.15)$ & $0.00(1.05)$ \\
\hline UGC9560 & 0.74 & 0.07 & -2.04 & -2.33 & $1.88 \mathrm{E}-11$ & $6.73 \mathrm{E}-11$ & $1.17 \mathrm{E}-10$ & 0.56 & 1.50 \\
\hline NGC5860 & 0.82 & 0.35 & -0.98 & -2.36 & $4.26 \mathrm{E}-11$ & $3.99 \mathrm{E}-11$ & $3.05 \mathrm{E}-10$ & 0.56 & 3.10 \\
\hline UGCA410 & 0.81 & 0.06 & -1.15 & -1.67 & $1.13 \mathrm{E}-11$ & $2.72 \mathrm{E}-11$ & $4.09 \mathrm{E}-11$ & 0.86 & $0.66 \mathrm{~L}$ \\
\hline NGC5996 & 0.38 & 0.23 & -0.99 & -2.03 & $4.04 \mathrm{E}-11$ & $6.08 \mathrm{E}-11$ & $2.72 \mathrm{E}-10$ & 0.21 & 0.98 \\
\hline NGC6052 & 0.89 & 0.10 & -0.87 & -1.32 & $2.32 \mathrm{E}-11$ & $6.91 \mathrm{E}-11$ & $1.24 \mathrm{E}-10$ & 0.08 & 0.21 \\
\hline NGC6090 & 0.96 & 0.30 & -0.78 & -2.11 & $7.03 \mathrm{E}-11$ & $4.42 \mathrm{E}-11$ & $2.85 \mathrm{E}-10$ & 0.21 & 1.42 \\
\hline NGC6217 & 0.20 & 0.27 & -0.61 & -1.75 & $6.35 \mathrm{E}-12$ & $1.07 \mathrm{E}-10$ & $5.06 \mathrm{E}-10$ & 0.01 & 0.64 \\
\hline Mrk499 & 1.03 & 0.22 & -1.02 & -1.93 & $9.52 \mathrm{E}-12$ & $3.91 \mathrm{E}-11$ & $1.69 \mathrm{E}-10$ & 0.18 & 1.46 \\
\hline Tol1924-416 & 0.93 & 0.01 & -2.08 & -2.15 & $3.67 \mathrm{E}-11$ & $1.70 \mathrm{E}-10$ & $1.81 \mathrm{E}-10$ & 0.42 & $\ldots$ \\
\hline $1941-543$ & 1.04 & 0.08 & -1.38 & -1.85 & $1.10 \mathrm{E}-11$ & $3.91 \mathrm{E}-11$ & $5.98 \mathrm{E}-11$ & 1.75 & $0.22 \mathrm{~L}$ \\
\hline NGC7250 & 0.26 & 0.05 & -1.47 & -1.56 & $2.16 \mathrm{E}-11$ & $1.12 \mathrm{E}-10$ & $1.46 \mathrm{E}-10$ & 0.12 & 0.39 \\
\hline NGC7552 & 0.33 & 0.35 & +0.43 & -1.04 & $2.76 \mathrm{E}-10$ & $3.16 \mathrm{E}-10$ & $1.26 \mathrm{E}-09$ & 0.07 & 0.43 \\
\hline NGC7673 & 0.48 & 0.21 & -1.48 & -2.38 & $4.34 \mathrm{E}-11$ & $1.22 \mathrm{E}-10$ & $4.25 \mathrm{E}-10$ & 0.17 & 2.17 \\
\hline NGC7714 & 0.82 & 0.20 & -1.23 & -2.02 & $1.83 \mathrm{E}-10$ & $2.15 \mathrm{E}-10$ & $6.42 \mathrm{E}-10$ & 0.31 & 3.28 \\
\hline
\end{tabular}

NoTES. $-L_{B}^{I U E} / L_{B}\left(\right.$ col. [2]) is the fraction of the galaxy blue luminosity which is contained within the $I U E$-optical aperture. $E_{c}(B-V)$ (col. [3]) is the color excess of the stellar continuum as defined in eq. (5). $\beta$ and $\beta_{i}$ (cols. [4] and [5]) are the observed UV power-law index and the extinction-corrected UV power-law index, respectively. $F_{\text {ion }}$ (col. [6]) is the ionizing flux as derived from eqs. (10)-(11). $F_{(912-8000)}^{\text {obs }}$ (col. [7]) is the integrated nonionizing flux obtained from the observed spectra in the wavelength range $912-8000 \AA$ (see $\S 4) . F_{(912-8000)}^{i}$ (col. [8]) is the integrated nonionizing flux obtained from the extinction-corrected spectra in the wavelength range $912-8000 \AA . f^{w} F_{\text {ion }} /$ FIR $^{w}$ (col. [9]) is the predicted-to-observed warm dust emission ratio. $f^{c} \delta F_{(912-8000)} /$ FIR $^{c}$ (col. [10]) is the predicted-to-observed cool dust emission ratio. An $L$ after a value denotes a lower limit.

Haro 15, NGC 1705, Mrk 66, and NGC 5253 have two sets of values for the intrinsic ionizing and nonionizing fluxes (cols. [6], [8], [9], and [10]). The first set is given by $E_{l}(B-V)=E_{c}(B-V)=0$, which is the color excess as calculated from the observed Balmer decrement. The second set of values, given in parentheses, corresponds to the ionizing and nonionizing fluxes as obtained by adopting the color excesses $E_{l}(B-V)=0.1$ and $E_{c}(B-V)=0.05$, respectively (see $\left.\S 4\right)$; for NGC $1705 E_{c}(B-V)=0.01\left(E_{l}[B-V]=0.02\right)$ is adopted, since this value of the color excess is enough to explain almost entirely the cool dust FIR emission.

4. Taking into account the uncertainties on the FIR flux, on the UV and optical spectra, on the obscuration curve, and on the adopted parameters, such as $T_{c}$ and the fraction $f_{\text {sg }}$ of the GISRF which heats the small grains/large molecules, the error bar on the ratio $f^{c} \delta F_{(912-8000)} / \mathrm{FIR}^{c}$ is of the order of $50 \%$, not incompatible with the observed spread in the data points.

The effect of the aperture mismatch between the UV-optical and FIR data on the data in Figure 11 can be investigated by plotting $f^{w} F_{\text {ion }} / \mathrm{FIR}^{w}$ and $f^{c} \delta F_{(912-8000)} / \mathrm{FIR}^{c}$ as a function of the fraction of the total blue luminosity encircled by the $I U E$ optical aperture, $L_{B(I U E)} / L_{B}$ (Figs. $12 a$ and $12 b$, respectively; see $\S 2.1)$. The implicit assumption here is that the spatial distribution of the FIR emission is, at most, as much extended as the blue light; this hypothesis is reasonable for galaxies. A weak increase of the predicted-to-observed warm dust emission is present for increasing fractions of the blue luminosity contained within the $I U E$ optical aperture. For instance, galaxies with $L_{B(I U E)} / L_{B} \geq 0.6$ have a median value $f^{w} F_{\text {ion }} / \mathrm{FIR}^{w} \simeq 0.3$.
A weak trend is also observed for the predicted-to-observed cool dust emission (Fig. 12b), and galaxies with $L_{B(I U E)}-L_{B} \geq$ 0.6 have median value $f^{c} \delta F_{(912-8000)} / \mathrm{FIR}^{c} \simeq 1.4$.

There is no correlation between $f^{c} \delta F_{(912-8000)} / \mathrm{FIR}^{c}$ and $E_{l}(B-V)$ (Fig. 13); therefore, the cool dust FIR emission increases with the nonionizing radiation lost to dust obscuration and with the observed color excess. The more obscured the galaxy, the larger the amount of FIR ${ }^{c}$ measured from the IRAS data.

The median $f^{w} F_{\text {ion }} / \mathrm{FIR}^{w} \simeq 0.3$ may still be an underestimated value for some of the galaxies: the ionizing flux $F_{\text {ion }}$ is calculated from the $\mathrm{H} \alpha$ flux in the $I U E$-optical aperture, which is in general smaller than the size of the galaxy. However, some considerations suggest that, at least for the galaxies with $60 \%$ or more of the total blue light within the $I U E$-optical aperture, the underestimation in the value of $F_{\text {ion }}$ is not large. Twophoton processes have not been included in the evaluation of $L_{\text {ion }}$ in equation (10), which already gives an overestimation of 

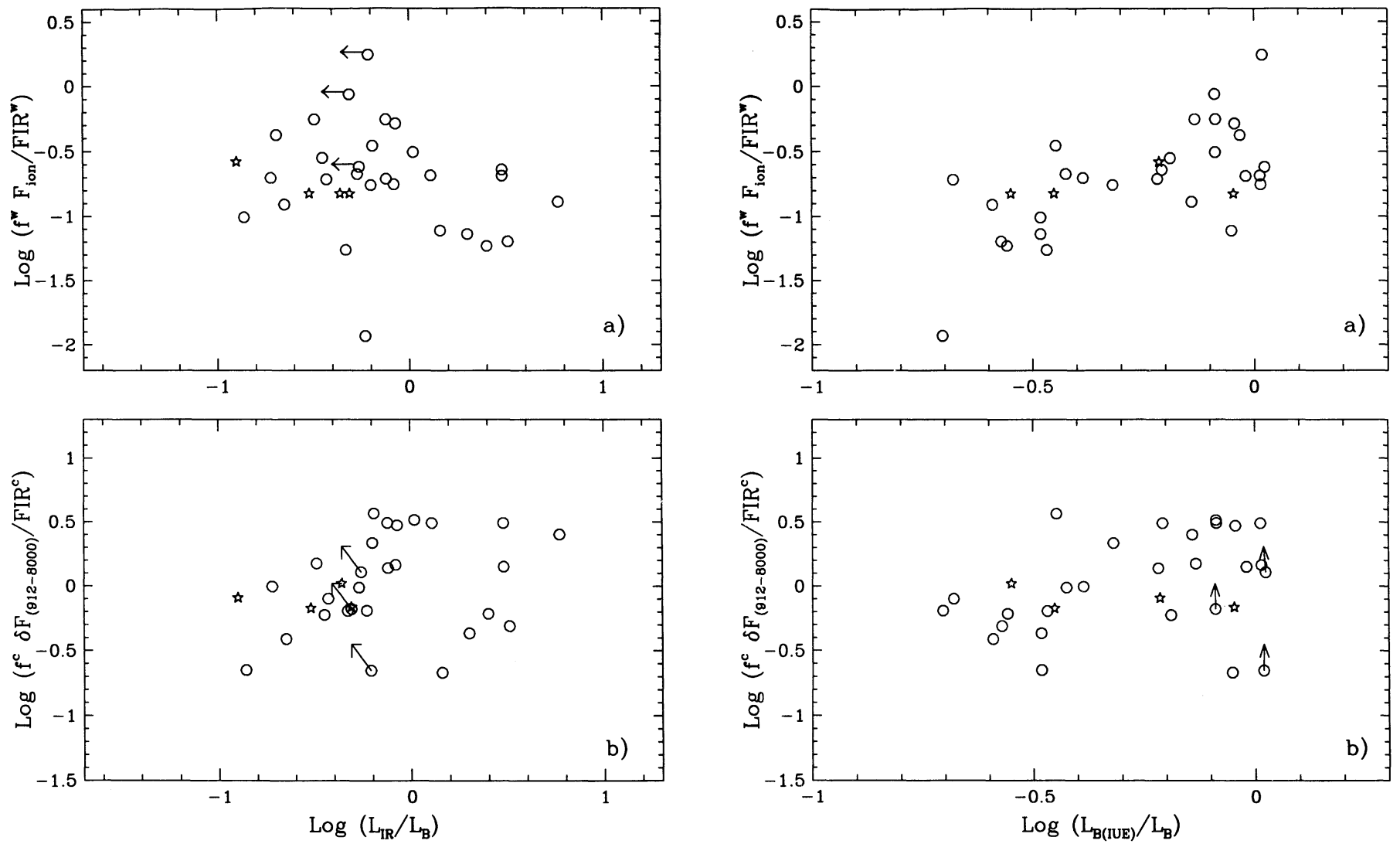

Fig. 11. $-(a)$ The predicted-to-observed warm dust FIR emission as a function of $\log \left(L_{\mathrm{IR}} / L_{B}\right)$. The ionizing radiation contributes only $20 \%-30 \%$ of the warm dust FIR emission. The stars indicate the four galaxies (NGC 1705, NGC 5253, Mrk 66, and Haro 15) for which an upper limit on the color excess $E_{l}(B-V)$ is given (see text). Arrows indicate upper limits on the FIR luminosity (UGCA 410, 1941-543, Mrk 542). (b) The predicted-to-observed cool dust FIR emission as a function of $\log \left(L_{\mathrm{IR}} / L_{B}\right)$. The median value is about 1 , meaning that the observed UV and optical radiation accounts for most of the cool dust emission. Upper limits on $L_{\mathrm{IR}}$ are assumed to be upper limits on FIR $^{c}$, but not on FIR ${ }^{w}$. Indeed, all galaxies in S2 are detected at $60 \mu \mathrm{m}$, which is where the warm dust emission peaks. UGC 410, 1941-543, and Mrk 542 have upper limits in the $100 \mu \mathrm{m}$ band, which is close to the peak of emission of the cool dust.

$L_{\text {ion }}$ of about $20 \%-25 \%$. In addition, no contribution of $L_{\text {ion }}$ to the heating of small grains has been considered, which carries another $20 \%$ overestimation in the value of the predicted warm dust emission $f^{w} F_{\text {ion }}$ (e.g., Xu et al. 1994). In summary, the values of $f^{w} F_{\text {ion }} / \mathrm{FIR}^{w}$ shown in Figures $11 a$ and $12 a$ are not far from the correct values.

The assumption that the entire nonionizing UV flux is associated with the GISRF and the cool dust emission must be relaxed for a detailed evaluation of the energy balance between UV and optical light lost to dust obscuration and FIR dust emission. The impact of the nonionizing radiation on the warm dust emission is determined by the assumption on the intrinsic ratio between the ionizing and the bolometric flux of the massive stars produced by the burst. As an example, we assume that the high-mass star population has a bolometric flux about 4 times the ionizing flux (the typical range is 2.5 to 7 ). The observed IUE spectrum is given by two contributions: the high-mass star population, affected by a color excess $E_{l}(B-V)$, and the general population, affected by a color excess $E_{c}(B-V)$. The median value of the predicted-to-observed cool dust emission then decreases from 1.4 to about 1 , for galaxies

FIG. 12.- (a) The predicted-to-observed warm dust FIR emission as a function of the fraction of blue light of the galaxy contained with the IUE aperture. Symbols are as in Fig. 11. (b) The predicted-to-observed cool dust FIR radiation as a function of the fraction of blue light contained within the IUE aperture.

with $L_{B(I U E)} / L_{B} \geq 0.6$. For the same galaxies, the median value of the predicted-to-observed warm dust emission increases from 0.3 to 0.7 .

The main conclusion from this section is that the amount of ionizing and nonionizing radiation lost to dust obscuration which can be extrapolated from the observed spectra is suffi-

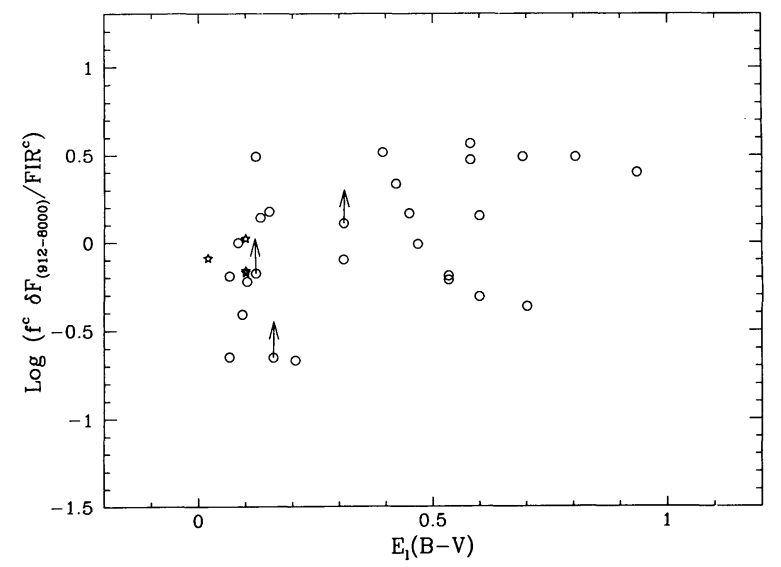

FIG. 13.-The predict-to-observed cool dust FIR radiation as a function of the color excess $E_{l}(B-V)$. Symbols are as in Fig. $11 b$. There is no variation of the predicted-to-observed cool dust FIR emission ratio for increasing values of the color excess. 
cient to account for the entire cool dust FIR emission and for about $70 \%$ of the warm dust FIR emission.

\section{SUMMARY AND CONCLUSIONS}

The relationship between dust absorption at UV and optical wavelengths and dust emission at infrared wavelengths has been investigated for a sample of starburst and blue compact galaxies.

The correlation between the UV spectral index $\beta$ and the FIR-to-blue ratio $L_{\mathrm{IR}} / L_{B}$, found in the present work, indicates that the stellar populations responsible for the observed UV and optical emission heat the dust which emits in the IRAS bands. An increase in dust obscuration has the multiple effect of reddening the UV spectral index, of decreasing the blue luminosity and of increasing the FIR luminosity (see eq. [15] and Fig. 10). The correlation between $\beta$ and $L_{\mathrm{IR}} / L_{B}$ cannot be completely explained unless an obscuration-dependent contribution to the FIR emission from the nonionizing radiation is included in the model ( $\$ 3.5)$.

The correlation between dust obscuration and the FIR-toblue ratio has not been observed in previous works (e.g., Salzer \& MacAlpine 1988). Part of the reason may reside in a possibly larger range of SFRs spanned by the galaxies included in other samples; and part may be in the use of the color excess $E(B-V)$ for quantifying the dust obscuration, since $E(B-V)$ has a smaller dynamical range than the UV spectral index $\beta$ used here (see eq. [2]).

About $70 \%$ of the FIR emission from our galaxies can be attributed to warm dust, with a range from $50 \%$ to $100 \%$, in agreement with the fact that the galaxies in the sample are actively forming stars and have a radiation field dominated by the emission from young stars. However, since $30 \%$ of the FIR emission is from cool dust, the GISRF is not negligible, even in galaxies undergoing strong star formation.

Previous attempts to determine the energy balance between the cool FIR emission and the fraction of the GISRF absorbed by dust have given uncertain results, mostly because of the incomplete information available at UV wavelengths and because an appropriate obscuration curve for extended regions was not available (LH; Hunter et al. 1989a; Xu 1990).

Using UV and optical spectra of the central regions of the galaxies and the obscuration curve derived by CKS, we have verified that the fraction of the GISRF lost to dust obscuration accounts entirely for the cool dust emission in our sample. The UV and optically detectable general stellar populations are sufficient to explain the emission from dust heated to $T \sim 20$ $\mathrm{K}$. The fraction of nonionizing stars embedded in dusty regions is, therefore, small (see $\mathrm{Xu} \mathrm{1990).} \mathrm{This} \mathrm{result} \mathrm{is} \mathrm{in} \mathrm{agreement}$ with the fact that long-lived stellar populations have had enough time to drift away from the dusty parent cloud and are located in regions of low dust content (Garmany et al. 1982).

The warm dust emission, which is attributed to the dust heating by high-mass star stars, is about a factor 1.5 higher than expected from the detailed energy balance (see $\S 4$ ). About $30 \%$ of the warm dust emission is due to massive stars which are embedded in dusty regions and are not manifest through UV or optical emission (Caux et al. 1984; LH; Hunter et al. 1989a).

This scenario is in general agreement with the result by CKS, who find that the dust obscuration affecting the general stellar population is smaller than the dust obscuration affecting the high-mass star population.

In normal disk galaxies, between $50 \%$ and $80 \%$ of the FIR emission, depending on the morphological type, is due to cool dust (Sauvage \& Thuan 1992); in actively star-forming galaxies this fraction is down to about $30 \%$, as expected for systems dominated by newly born stars. The presence of cool dust emission implies that FIR $^{c}$ must be removed if the FIR emission is used as an indicator of high-mass SFR.

The authors thank Rosemary Wyse, Claus Leitherer, and Mauro Giavalisco for suggestions and useful discussions. We thank an anonymous referee for the many useful comments which have largely improved the presentation of this work.

The authors acknowledge support from the NASA grant NAG5-1143 and from the STScI Director Discretionary Research Fund.

T. S. B. acknowledges support from the Brazilian Institutions $\mathrm{CNP}_{q}, \mathrm{CAPES}$, and FAPERGS.

This research has made use of the NASA/IPAC Extragalactic Database (NED) which is operated by the Jet Propulsion Laboratory, Caltech, under contract with the National Aeronautics and Space Administration.
Andriesse, C. D. 1978, A\&A, 66, 169

Belfort, P., Mochkovitch, R., \& Dennefeld, M. 1987, A\&A, 176, 1

Belley, J., \& Roy, J.-R. 1992, ApJS, 78, 61

Boulanger, F., Beichman, C., Désert, F. X., Helou, G., Pérault, M., \& Ryter, C. 1988, ApJ, 332, 328

Boulanger, F., \& Pérault, M. 1988, ApJ, 330, 694

Buat, V., \& Deharveng, J. M. 1988, A\&A, 195, 60

Calzetti, D., Kinney, A. L., \& Storchi-Bergmann, T. 1994, ApJ, 429, 582 (CKS)

Caux, E., Serra, G., Gispert, R., Puget, J. L., Ryter, C., \& Coron, N. 1984, A\&A, 137,1

Chlewicki, G., \& Greenberg, J. M. 1990, ApJ, 365, 230

Chini, R., Kreysa, E., Krügel, E., \& Mezger, P. G. 1986, A\&A, 166, L8

Cox, P., Krügel E., \& Mezger, P. G. 1986, A\&A, 155, 380

de Jong, T., \& Brink, K. 1987, Star Formation in Galaxies, ed. C. J. LonsdalePersson (NASA-CP 2466), 323

de Jong, T., Clegg, P. E., Soifer, B. T., Rowan-Robinson, M., Habing, H. J., Houck, J. R., Aumann, H. H., \& Raimond, E. 1984, ApJ, 278, L67

Désert, F.-X. 1986, Light on Dark Matter, ed. F. P. Israel (Dordrecht: Reidel), 213

Désert, F.-X., Boulanger, F., \& Puget, J. L. 1990, A\&A, 237, 215

Devereux, N. A., \& Young, J. S. 1991, ApJ, 371, 515 1992, AJ, 103, 1536 1993, AJ, 106, 948

Draine, B. T. 1990, The Interstellar Medium in Galaxies, ed. H. A. Thronson \& J. M. Shull (Dordrecht: Kluwer), 483

\section{REFERENCES}

Draine, B. T., \& Anderson, N. 1985, ApJ, 292, 494

Duley, W. W., Jones, A. P., \& Williams, D. A. 1989, MNRAS, 236, 709

Fabbiano, G., \& Trinchieri, G. 1987, ApJ, 315, 46

Fanelli, M. N., O'Connell, R. W., \& Thuan, T. X. 1988, ApJ, 334, 665

Fullmer, L., \& Lonsdale, C. J. 1989, Cataloged Galaxies and Quasars Observed in the IRAS Survey, Version 2

Garmany, C. D., Conti, P. S., \& Chiosi, C. 1982, ApJ, 263, 777

Helou, G. 1986, ApJ, 311, L33

Helou, G., Khan, I. R., Malek, L., \& Boehmer, L. 1988, ApJS, 68, 151

Helou, G., Soifer, B. T., \& Rowan-Robinson, M. 1985, ApJ, 298, L7

Hunter, D. A., Gallagher, J. S., Rice, W. L., \& Gillett, F. C. 1989a, ApJ, 336, 152

Hunter, D. A., Thronson, H. A., Casey, S., \& Harper, D. A. 1989b, ApJ, 341, 697

Joy, M., Lester, D. F., Harvey, P. M., Telesco, C. M., Decher, R., Rickard, L. J., \& Bushouse, H. 1989, ApJ, 339, 100

Keel, W. C. 1993, Massive Stars: Their Lives in the Interstellar Medium, ed. J. P. Cassinelli \& E. B. Churchwell (ASP Conf. Ser., 35), 498

Kennicutt, R. C. 1983, ApJ, 272, 54

Kennicutt, R. C., Keel, W. C., van der Hulst, J. M., Hummel, E., \& Roettiger, K. A. 1987, AJ, 93, 1011

Kinney, A. L., Bohlin, R. C., Calzetti, D., Panagia, N., \& Wyse, R. F. G. 1993, ApJS, 86, 5

Kinney, A. L., Calzetti, D., Bica, E. L., \& Storchi-Bergmann, T. 1994, ApJ, 429,

Kwan, J., \& Xie, S. 1992, ApJ, 398, 105 
Léger, A., \& Puget, J. L. 1984, A\&A, 137, L5

Leitherer, C., \& Heckman, T. M. 1994, ApJ, 428, 292

Lequeux, J. 1980, Star Formation, 10th Advanced Course, Swiss Society of Astronomy and Astrophysics, ed. A. Maeder \& L. Martinet (Geneva: Geneva Obs.), 75

I Lonsdale-Persson, C. J., \& Helou, G. 1987, ApJ, 314, 513 (LH)

Low, F. J., et al. 1984, ApJ, 278, L19

Martin, P., \& Roy, J.-R. 1992, ApJ, 397, 463

Mathis, J. S., \& Whiffen, G. 1989, ApJ, 341, 808

Mezger, P. G. 1978, A\&A, 70, 565

Mezger, P. G., Mathis, J. S., \& Panagia, N. 1982, A\&A, 105, 372

Pajot, F., Boissé, P., Gispert, R., Lamarre, J. M., Puget, J. L., \& Serra, G. 1986, A\&A, 157, 393

Puget, J. L., \& Léger, A. 1989, ARA\&A, 27, 161

Rice, W. 1993, AJ, 105, 67

Rice, W., Lonsdale, C. J., Soifer, B. T., Neugebauer, G., Kopan, E. L., Lloyd, L. A., de Jong, T., \& Habing, H. J. 1988, ApJS, 68, 91

Rieke, G. H., Lebofsky, M. J., Thompson, R., Low, F., \& Tokunaga, A. 1980, ApJ, 238, 24
Rowan-Robinson, M., \& Crawford, J. 1987, Light on Dark Matter, ed. F. P. Israel (Dordrecht: Reidel), 421

Salzer, J. J., \& MacAlpine, G. M. 1988, AJ, 96, 1192

Sauvage, M., \& Thuan, T. X. 1992, ApJ, 396, L69

Seaton, M. J. 1979, MNRAS, 187, 73P

Sellegren, K. 1984, ApJ, 277, 623

Soifer, B. T., Boehmer, L., Neugebauer, G., \& Sanders, D. B. 1989, AJ, 98, 766 Surace, J. A., Mazzarella, J., Soifer, B. T., \& Wehrle, A. E. 1993, AJ, 105, 864

Smith, L. F., Biermann, P., \& Mezger, P. G. 1978, A\&A, 66, 65

Storchi-Bergmann, T., Calzetti, D., \& Kinney, A. L. 1994, ApJ, 429, 572

Telesco, C. M., Decher, R., \& Joy, M. 1989, 343, L13

Telesco, C. M., Dressler, L. L., \& Wolstencroft, R. D. 1993, ApJ, 414, 120

Thronson, H. A., Hunter, D. A., Casey, S., \& Harper, D. A. 1990, ApJ, 355, 94

van der Hulst, J. M., Kennicutt, R. C., Crane, P. C., \& Rots, A. H. 1988, A\&A, 195,38

Xu, C. 1990, ApJ, 365, L47

Xu, C., Lisenfeld, U., \& Völk, H. J. 1994, A\&A, in press

Young, J. S., Xie, S., Kenney, J. D. P., \& Rice, W. L. 1989, ApJS, 70, 699 